\title{
DERIVATIVE AND DIVERGENCE FORMULAE FOR DIFFUSION SEMIGROUPS $^{1}$
}

\author{
By ANTON Thalmaier AND JAMES THOMPSON \\ University of Luxembourg
}

\begin{abstract}
For a semigroup $P_{t}$ generated by an elliptic operator on a smooth manifold $M$, we use straightforward martingale arguments to derive probabilistic formulae for $P_{t}(V(f))$, not involving derivatives of $f$, where $V$ is a vector field on $M$. For nonsymmetric generators, such formulae correspond to the derivative of the heat kernel in the forward variable. As an application, these formulae can be used to derive various shift-Harnack inequalities.
\end{abstract}

Introduction. For a Banach space $E, e \in E$ and a Markov operator $P$ on $\mathcal{B}_{b}(E)$, it is known that certain estimates on $P\left(\nabla_{e} f\right)$ are equivalent to corresponding shift-Harnack inequalities. This was proved by F.-Y. Wang in [18]. For example, for $\delta_{e} \in(0,1)$ and $\beta_{e} \in C\left(\left(\delta_{e}, \infty\right) \times E ;[0, \infty)\right)$, he proved that the derivativeentropy estimate

$$
\left|P\left(\nabla_{e} f\right)\right| \leq \delta(P(f \log f)-(P f) \log P f)+\beta_{e}(\delta, \cdot) P f
$$

holds for any $\delta \geq \delta_{e}$ and positive $f \in C_{b}^{1}(E)$ if and only if the inequality

$$
\begin{aligned}
(P f)^{p} \leq & \left(P\left(f^{p}(r e+\cdot)\right)\right) \\
& \times \exp \left(\int_{0}^{1} \frac{p r}{1+(p-1) s} \beta_{e}\left(\frac{p-1}{r+r(p-1) s}, \cdot+s r e\right) d s\right)
\end{aligned}
$$

holds for any $p \geq 1 /\left(1-r \delta_{e}\right), r \in\left(0,1 / \delta_{e}\right)$ and positive $f \in \mathcal{B}_{b}(E)$. Furthermore, he also proved that if $C \geq 0$ is a constant then the $L^{2}$-derivative inequality

$$
\left|P\left(\nabla_{e} f\right)\right|^{2} \leq C P f^{2}
$$

holds for any nonnegative $f \in C_{b}^{1}(E)$ if and only if the inequality

$$
P f \leq P(f(\alpha e+\cdot))+|\alpha| \sqrt{C P f^{2}}
$$

holds for any $\alpha \in \mathbb{R}$ and nonnegative $f \in \mathcal{B}_{b}(E)$. The objective of this article is to find probabilistic formulae for $P_{T}(V(f))$ from which such estimates can be

Received January 2017; revised February 2018.

${ }^{1}$ Supported by the Fonds National de la Recherche Luxembourg (FNR) under the OPEN scheme (project GEOMREV O14/7628746).

MSC2010 subject classifications. Primary 58J65, 60J60; secondary 53C21.

Key words and phrases. Diffusion semigroup, heat kernel, gradient estimate, Harnack inequality, Ricci curvature. 
derived, for the case in which $P_{T}$ is the Markov operator associated to a nondegenerate diffusion $X_{t}$ on a smooth, finite-dimensional manifold $M$, and $V$ a vector field.

In Section 1, we suppose that $M$ is a Riemannian manifold and that the generator of $X_{t}$ is $\Delta+Z$, for some smooth vector field $Z$. Any nondegenerate diffusion on a smooth manifold induces a Riemannian metric with respect to which its generator takes this form. The basic strategy is then to use the relation $V(f)=\operatorname{div}(f V)-$ $f \operatorname{div} V$ to reduce the problem to finding a suitable formula for $P_{T}(\operatorname{div}(f V))$. Such formulae have been given in [3] and [8] for the case $Z=0$, which we extend to the general case with Theorem 1.16. In doing so, we do not make any assumptions on the derivatives of the curvature tensor, as occurred in [2]. For an adapted process $h_{t}$ with paths in the Cameron-Martin space $L^{1,2}([0, T] ; \mathbb{R})$, with $h_{0}=0$ and $h_{T}=1$ and under certain additional conditions, we obtain the formula

$$
\begin{aligned}
P_{T}(V(f))(x) \\
=-\mathbb{E}\left[f\left(X_{T}(x)\right)(\operatorname{div} V)\left(X_{T}(x)\right)\right] \\
\quad+\frac{1}{2} \mathbb{E}\left[f\left(X_{T}(x)\right)\left\langle V\left(X_{T}(x)\right), / /_{T} \Theta_{T} \int_{0}^{T}\left(\dot{h}_{t}-(\operatorname{div} Z)\left(X_{t}(x)\right) h_{t}\right) \Theta_{t}^{-1} d B_{t}\right\rangle\right]
\end{aligned}
$$

where $\Theta$ is the $\operatorname{Aut}\left(T_{x} M\right)$-valued process defined by the pathwise differential equation

$$
\frac{d}{d t} \Theta_{t}=-/ /_{t}^{-1}\left(\operatorname{Ric}^{\sharp}+(\nabla \cdot Z)^{*}-\operatorname{div} Z\right) / /_{t} \Theta_{t}
$$

with $\Theta_{0}=\mathrm{id}_{T_{x} M}$. Here, $/ / t$ denotes the stochastic parallel transport associated to $X_{t}(x)$, whose antidevelopment to $T_{x} M$ has martingale part $B$. In particular, $B$ is a diffusion on $\mathbb{R}^{n}$ generated by the Laplacian; it is a standard Brownian motion sped up by 2 , so that $d B_{t}^{i} d B_{t}^{j}=2 \delta_{i j} d t$. Choosing $h_{t}$ explicitly yields a formula from which estimates then can be deduced, as described in Section 1.5.

The problem of finding a suitable formula for $P_{T}(V(f))$ is dual to that of finding an analogous one for $V\left(P_{T} f\right)$. A formula for the latter is called the Bismut formula [1] or the Bismut-Elworthy-Li formula, on account of [7]. We provide a brief proof of it in Section 1.3, since we would like to compare it to our formula for $P_{T}(V(f))$. Our approach to these formulae is based on martingale arguments; integration by parts is done at the level of local martingales. Under conditions which assure that the local martingales are true martingales, the wanted formulae are then obtained by taking expectations. They allow for the choice of a finite energy process. Depending on the intended type, conditions are imposed either on the right endpoint, as in the formula for $P_{T}(V(f))$, or the left endpoint, as in the formula for $V\left(P_{T} f\right)$. The formula for $P_{T}(V(f))$ requires nonexplosivity; the formula for $V\left(P_{T} f\right)$ does not. From the latter can be deduced Bismut's formula for 
the logarithmic derivative in the backward variable $x$ of the heat kernel $p_{T}(x, y)$ determined by

$$
\left(P_{T} f\right)(x)=\int_{M} f(y) p_{T}(x, y) \operatorname{vol}(d y), \quad f \in C_{b}(M) .
$$

From our formula for $P_{T}(V(f))$ can be deduced the following formula for the derivative in the forward variable $y$ :

$$
\begin{aligned}
& \left(\nabla \log p_{T}(x, \cdot)\right)_{y} \\
& \quad=-\frac{1}{2} \mathbb{E}\left[/ / \Theta_{T} \int_{0}^{T}\left(\dot{h}_{t}-(\operatorname{div} Z)\left(X_{t}(x)\right) h_{t}\right) \Theta_{t}^{-1} d B_{t} \mid X_{T}(x)=y\right] .
\end{aligned}
$$

In Section 2, we consider the general case in which $M$ is a smooth manifold and $X_{t}$ a nondegenerate diffusion solving a Stratonovich equation of the form

$$
d X_{t}=A_{0}\left(X_{t}\right) d t+A\left(X_{t}\right) \circ d B_{t} .
$$

We denote by $T X_{t}$ the derivative (in probability) of the solution flow. Using a similar approach to that of Section 1 , and a variety of geometric objects naturally associated to the equation, we obtain, under certain conditions, the formula

$$
\begin{aligned}
& P_{T}(V(f)) \\
& =-\sum_{i=1}^{m} \mathbb{E}\left[f\left(X_{T}\right) A_{i}\left\langle V\left(X_{T}, A_{i}\right\rangle\left(X_{T}\right)\right]\right. \\
& \quad+\frac{1}{2} \mathbb{E}\left[f ( X _ { T } ) \left\langleV\left(X_{T}\right), \Xi_{T} \int_{0}^{T} \Xi_{t}^{-1}\left(\left(\dot{h}_{t}-\left(\operatorname{trace} \hat{\nabla} A_{0}\right)\left(X_{t}\right) h_{t}\right) A\left(X_{t}\right) d B_{t}\right.\right.\right. \\
& \left.\left.\left.\quad+2 h_{t} A_{0}^{A} d t\right)\right\rangle\right]
\end{aligned}
$$

with

$$
\begin{aligned}
\Xi_{t} & =T X_{t}-T X_{t} \int_{0}^{t} T X_{s}^{-1}\left(\left(\left(\hat{\nabla} A_{0}\right)^{*}+\hat{\nabla} A_{0}+\operatorname{trace} \hat{\nabla} A_{0}\right)\left(\Xi_{s}\right)\right) d s, \\
A_{0}^{A} & =\sum_{i=1}^{m}\left(\left(\hat{\nabla} A_{0}\right)^{*}+\hat{\nabla} A_{0}\right)\left(\breve{T}\left(\cdot, A_{i}\right)^{*}\left(A_{i}\right)\right)+\left[A_{0}, \breve{T}\left(\cdot, A_{i}\right)^{*}\left(A_{i}\right)\right],
\end{aligned}
$$

where the operators $\hat{\nabla} A_{0}$ and $\breve{T}\left(\cdot, A_{i}\right)$ are given at each $x \in M$ and $v \in T_{x} M$ by

$$
\begin{aligned}
\hat{\nabla}_{v} A_{0} & =A(x)\left(d\left(A^{*}(\cdot) A_{0}(\cdot)\right)_{x}(v)-\left(d A^{*}\right)_{x}\left(v, A_{0}\right)\right), \\
\breve{T}\left(v, A_{i}\right)_{x} & =A(x)\left(d A^{*}\right)_{x}\left(v, A_{i}\right) .
\end{aligned}
$$

This formula has the advantage of involving neither parallel transport nor Riemannian curvature, both typically difficult to calculate in terms of $A$. 


\section{Intrinsic formulae.}

1.1. Preliminaries. Let $M$ be a complete and connected $n$-dimensional Riemannian manifold, $\nabla$ the Levi-Civita connection on $M$ and $\pi: \mathrm{O}(M) \rightarrow M$ the orthonormal frame bundle over $M$. Let $E \rightarrow M$ be an associated vector bundle with fibre $V$ and structure group $G=\mathrm{O}(n)$. The induced covariant derivative

$$
\nabla: \Gamma(E) \rightarrow \Gamma\left(T^{*} M \otimes E\right)
$$

determines the so-called connection Laplacian (or rough Laplacian) $\square$ on $\Gamma(E)$,

$$
\square a=\operatorname{trace} \nabla^{2} a .
$$

Note that $\nabla^{2} a \in \Gamma\left(T^{*} M \otimes T^{*} M \otimes E\right)$ and $(\square a)_{x}=\sum_{i} \nabla^{2} a\left(v_{i}, v_{i}\right) \in E_{x}$ where $v_{i}$ runs through an orthonormal basis of $T_{x} M$. For $a, b \in \Gamma(E)$ of compact support it is immediate to check that

$$
\langle\square a, b\rangle_{L^{2}(E)}=-\langle\nabla a, \nabla b\rangle_{L^{2}\left(T^{*} M \otimes E\right)} .
$$

In this sense, we have $\square=-\nabla^{*} \nabla$. Let $H$ be the horizontal subbundle of the $G$ invariant splitting of $T \mathrm{O}(M)$ and

$$
h: \pi^{*} T M \stackrel{\sim}{\longrightarrow} H \hookrightarrow T \mathrm{O}(M)
$$

the horizontal lift of the $G$-connection; fiberwise this bundle isomorphism reads as

$$
h_{u}: T_{\pi(u)} M \stackrel{\sim}{\longrightarrow} H_{u}, \quad u \in \mathrm{O}(M) .
$$

In terms of the standard horizontal vector fields $H_{1}, \ldots, H_{n}$ on $\mathrm{O}(M)$,

$$
H_{i}(u):=h_{u}\left(u e_{i}\right), \quad u \in \mathrm{O}(M),
$$

Bochner's horizontal Laplacian $\Delta^{\text {hor }}$, acting on smooth functions on $\mathrm{O}(M)$, is given as

$$
\Delta^{\text {hor }}=\sum_{i=1}^{n} H_{i}^{2} .
$$

To formulate the relation between $\square$ and $\Delta^{\text {hor }}$, it is convenient to write sections $a \in \Gamma(E)$ as equivariant functions $F_{a}: \mathrm{O}(M) \rightarrow V$ via $F_{a}(u)=u^{-1} a_{\pi(u)}$ where we read $u \in \mathrm{O}(M)$ as an isomorphism $u: V \stackrel{\sim}{\longrightarrow} E_{\pi(u)}$. Equivariance means that

$$
F_{a}(u g)=g^{-1} F_{a}(u), \quad u \in \mathrm{O}(M), g \in G=\mathrm{O}(n) .
$$

LEMMA 1.1 (See [10], page 115). For $a \in \Gamma(E)$ and $F_{a}$ the corresponding equivariant function on $\mathrm{O}(M)$, we have

$$
\left(H_{i} F_{a}\right)(u)=F_{\nabla_{u e_{i}} a}(u), \quad u \in \mathrm{O}(M) .
$$


Hence

$$
\Delta^{\text {hor }} F_{a}=F_{\square a}
$$

where as above

$$
\square: \Gamma(E) \stackrel{\nabla}{\longrightarrow} \Gamma\left(T^{*} M \otimes E\right) \stackrel{\nabla}{\longrightarrow} \Gamma\left(T^{*} M \otimes T^{*} M \otimes E\right) \stackrel{\text { trace }}{\longrightarrow} \Gamma(E) .
$$

Proof. Fix $u \in \mathrm{O}(M)$ and choose a curve $\gamma$ in $M$ such that $\gamma(0)=\pi(u)$ and $\dot{\gamma}=u e_{i}$. Let $t \mapsto u(t)$ be the horizontal lift of $\gamma$ to $\mathrm{O}(M)$ such that $u(0)=u$. Note that $\dot{u}(t)=h_{u(t)}(\dot{\gamma}(t))$, and in particular $\dot{u}(0)=h_{u}\left(u e_{i}\right)=H_{i}(u)$. Hence, denoting the parallel transport along $\gamma$ by $/ /_{\varepsilon}=u(\varepsilon) u(0)^{-1}$, we get

$$
\begin{aligned}
F_{\nabla_{u e_{i}} a}(u) & =u^{-1}\left(\nabla_{u e_{i}} a\right)_{\pi(u)} \\
& =u^{-1} \lim _{\varepsilon \downarrow 0} \frac{/ /_{\varepsilon}^{-1} a_{\gamma(\varepsilon)}-a_{\gamma(0)}}{\varepsilon} \\
& =\lim _{\varepsilon \downarrow 0} \frac{u(\varepsilon)^{-1} a_{\gamma(\varepsilon)}-u(0)^{-1} a_{\gamma(0)}}{\varepsilon} \\
& =\lim _{\varepsilon \downarrow 0} \frac{F_{a}(u(\varepsilon))-F_{a}(u(0))}{\varepsilon} \\
& =\left(H_{i}\right)_{u} F_{a} \\
& =\left(H_{i} F_{a}\right)(u) .
\end{aligned}
$$

Now consider diffusion processes $X_{t}$ on $M$ generated by the operator

$$
\mathscr{L}=\Delta+Z,
$$

where $Z \in \Gamma(T M)$ is a smooth vector field. Such diffusions on $M$ may be constructed from the corresponding horizontal diffusions on $\mathrm{O}(M)$ generated by

$$
\Delta^{\text {hor }}+\bar{Z} \text {, }
$$

where the vector field $\bar{Z}$ is the horizontal lift of $Z$ to $\mathrm{O}(M)$, that is, $\bar{Z}_{u}=$ $h_{u}\left(Z_{\pi(u)}\right), u \in \mathrm{O}(M)$. More precisely, we start from the Stratonovich stochastic differential equation on $\mathrm{O}(M)$,

$$
d U_{t}=\sum_{i=1}^{n} H_{i}\left(U_{t}\right) \circ d B_{t}^{i}+\bar{Z}\left(U_{t}\right) d t, \quad U_{0}=u \in \mathrm{O}(M),
$$

where $B_{t}$ is a Brownian motion on $\mathbb{R}^{n}$ sped up by 2 , that is $d B_{t}^{i} d B_{t}^{j}=2 \delta_{i j} d t$. Then for $X_{t}=\pi\left(U_{t}\right)$, the following equation holds:

$$
d X_{t}=\sum_{i=1}^{n} U_{t} e_{i} \circ d B_{t}^{i}+Z\left(X_{t}\right) d t, \quad X_{0}=x:=\pi u .
$$


The Brownian motion $B$ is the martingale part of the antidevelopment $\int_{U} \vartheta$ of $X$, where $\vartheta$ denotes the canonical 1-form $\vartheta$ on $\mathrm{O}(M)$, that is,

$$
\vartheta_{u}(e)=u^{-1} e_{\pi(u)}, \quad e \in T_{u} \mathrm{O}(M) .
$$

In particular, for $F \in C^{\infty}(\mathrm{O}(M))$, respectively, $f \in C^{\infty}(M)$, we have

$$
\begin{aligned}
d\left(F \circ U_{t}\right) & =\sum_{i=1}^{n}\left(H_{i} F\right)\left(U_{t}\right) \circ d B_{t}^{i}+(\bar{Z} F)\left(U_{t}\right) d t \\
& =\sum_{i=1}^{n}\left(H_{i} F\right)\left(U_{t}\right) d B_{t}^{i}+\left(\Delta^{\mathrm{hor}}+\bar{Z}\right)(F)\left(U_{t}\right) d t,
\end{aligned}
$$

respectively,

$$
\begin{aligned}
d\left(f \circ X_{t}\right) & =\sum_{i=1}^{n}(d f)\left(U_{t} e_{i}\right) \circ d B_{t}^{i}+(Z f)\left(X_{t}\right) d t \\
& =\sum_{i=1}^{n}(d f)\left(U_{t} e_{i}\right) d B_{t}^{i}+(\Delta+Z)(f)\left(X_{t}\right) d t .
\end{aligned}
$$

Typically, solutions to (1.2) are defined up to some maximal lifetime $\zeta(x)$ which may be finite. Then we have, almost surely,

$$
\{\zeta(x)<\infty\} \subset\left\{X_{t} \rightarrow \infty \text { as } t \uparrow \zeta(x)\right\},
$$

where on the right-hand side, the symbol $\infty$ denotes the point at infinity in the one-point compactification of $M$. It can be shown that the maximal lifetime of solutions to equation (1.1) and to (1.2) coincide; see, for example, [13].

In case of a nontrivial lifetime the subsequent stochastic equations should be read for $t<\zeta(x)$.

Proposition 1.2. Let $/ /_{t}: E_{X_{0}} \rightarrow E_{X_{t}}$ be parallel transport in $E$ along $X$, induced by the parallel transport on $M$,

$$
/_{t}=U_{t} U_{0}^{-1}: T_{X_{0}} M \rightarrow T_{X_{t}} M .
$$

Then, for $a \in \Gamma(E)$, we have

$$
d\left(/ /{ }_{t}^{-1} a\left(X_{t}\right)\right)=\sum_{i=1}^{n} / /_{t}^{-1}\left(\nabla_{U_{t} e_{i}} a\right) \circ d B_{t}^{i}+/ /{ }_{t}^{-1}\left(\nabla_{Z} a\right)\left(X_{t}\right) d t,
$$

respectively in Itô form,

$$
d\left(/ /\left.\right|_{t} ^{-1} a\left(X_{t}\right)\right)=\sum_{i=1}^{n} / /{ }_{t}^{-1}\left(\nabla_{U_{t} e_{i}} a\right) d B_{t}^{i}+/ / t_{t}^{-1}\left(\square a+\nabla_{Z} a\right)\left(X_{t}\right) d t .
$$


More succinctly, the last two equations may be written as

$$
d\left(/ / t^{-1} a\left(X_{t}\right)\right)=/ / t^{-1} \nabla_{\circ} X_{t} a,
$$

respectively,

$$
d\left(/ /_{t}^{-1} a\left(X_{t}\right)\right)=/ /_{t}^{-1} \nabla_{d X_{t}} \alpha+/ /_{t}^{-1}(\square a)\left(X_{t}\right) d t .
$$

Proof. We have $/ /_{t}^{-1} a\left(X_{t}\right)=U_{0} U_{t}^{-1} a\left(X_{t}\right)=U_{0} F_{a}\left(U_{t}\right)$. It is easily checked that $\bar{Z} F_{a}=F_{\nabla_{Z} a}$. Thus, we obtain from equation (1.3)

$$
\begin{aligned}
d F_{a}\left(U_{t}\right) & =\sum_{i=1}^{n}\left(H_{i} F_{a}\right)\left(U_{t}\right) d B_{t}^{i}+\left(\Delta^{\mathrm{hor}} F_{a}+\bar{Z} F_{a}\right)\left(U_{t}\right) d t \\
& =\sum_{i=1}^{n}\left(F_{\nabla_{U_{t} e_{i}} a}\right)\left(U_{t}\right) d B_{t}^{i}+\left(F_{\square a}+F_{\nabla_{Z} a}\right)\left(U_{t}\right) d t \\
& =\sum_{i=1}^{n} U^{-1}\left(\nabla_{U_{t} e_{i}} a\right)\left(X_{t}\right) d B_{t}^{i}+U_{t}^{-1}\left(\square a+\nabla_{Z} a\right)\left(X_{t}\right) d t .
\end{aligned}
$$

COROLlary 1.3. Fix $T>0$ and let $a_{t} \in \Gamma(E)$ solve the equation

$$
\frac{\partial}{\partial t} a_{t}=\square a_{t}+\nabla_{Z} a_{t} \quad \text { on }[0, T] \times M .
$$

Then

$$
/ /_{t}^{-1} a_{T-t}\left(X_{t}\right), \quad 0 \leq t<T \wedge \zeta(x),
$$

is a local martingale.

Proof. Indeed we have

$$
d\left(/ / /_{t}^{-1} a_{T-t}\left(X_{t}\right)\right) \stackrel{m}{=} / /_{t}^{-1} \underbrace{\left(\square a_{T-t}+\nabla_{Z} a_{t}+\frac{\partial}{\partial t} a_{T-t}\right)}_{=0}\left(X_{t}\right) d t=0,
$$

where $\stackrel{m}{=}$ denotes equality modulo differentials of local martingales.

We are now going to look at operators $\mathscr{L}^{\mathscr{R}}$ on $\Gamma(E)$ which differ from $\square$ by a zero-order term, in other words,

$$
\square-\mathscr{L}^{\mathscr{R}}=\mathscr{R} \quad \text { where } \mathscr{R} \in \Gamma(\text { End } E) .
$$

Thus, by definition, the action $\mathscr{R}_{x}: E_{x} \rightarrow E_{x}$ is linear for each $x \in M$. 
EXAMPLE 1.4. A typical example is $E=\Lambda^{p} T^{*} M$ and $A^{p}(M)=$ $\Gamma\left(\Lambda^{p} T^{*} M\right)$ with $p \geq 1$. The de Rham-Hodge Laplacian

$$
\Delta^{(p)}=-\left(d^{*} d+d d^{*}\right): A^{p}(M) \rightarrow A^{p}(M)
$$

then takes the form

$$
\Delta^{(p)} \alpha=\square \alpha-\mathscr{R} \alpha,
$$

where $\mathscr{R}$ is given by the Weitzenböck decomposition. In the special case $p=1$, one obtains $\mathscr{R} \alpha=\operatorname{Ric}\left(\alpha^{\sharp}, \cdot\right)$ where Ric: $T M \oplus T M \rightarrow \mathbb{R}$ is the Ricci tensor.

Definition 1.5. Fix $x \in M$ and let $X_{t}$ be a diffusion to $\mathscr{L}=\Delta+Z$, starting at $x$. Let $Q_{t}$ be the $\operatorname{Aut}\left(E_{x}\right)$-valued process defined by the following linear pathwise differential equation:

$$
\frac{d}{d t} Q_{t}=-Q_{t} \mathscr{R}_{/ / t}, \quad Q_{0}=\operatorname{id}_{E_{x}},
$$

where

$$
\mathscr{R}_{/ / t}:=/ / t^{-1} \circ \mathscr{R}_{X_{t}} \circ / / t \in \operatorname{End}\left(E_{x}\right)
$$

and $/ / t$ is parallel transport in $E$ along $X$.

Proposition 1.6. Let $\mathscr{L}^{\mathscr{R}}=\square-\mathscr{R}$ be as in equation (1.4) and $X_{t}$ be a diffusion to $\mathscr{L}=\Delta+Z$, starting at $x$. Then, for any $a \in \Gamma(E)$,

$$
\begin{aligned}
& d\left(Q_{t} / / t_{t}^{-1} a\left(X_{t}\right)\right) \\
& \quad=\sum_{i=1}^{n} Q_{t} / /_{t}^{-1}\left(\nabla_{U_{t} e_{i}} a\right) d B_{t}^{i}+Q_{t} / /_{t}^{-1}\left(\square a+\nabla_{Z} a-\mathscr{R} a\right)\left(X_{t}\right) d t .
\end{aligned}
$$

Proof. Let $n_{t}:=/ /_{t}^{-1} a\left(X_{t}\right)$. Then

$$
\begin{aligned}
d\left(Q_{t} n_{t}\right) & =\left(d Q_{t}\right) n_{t}+Q_{t} d n_{t} \\
& =-Q_{t} / /_{t}^{-1} \mathscr{R}_{X_{t}} / /_{t}^{-1} n_{t} d t+Q_{t} d n_{t} \\
& =-Q_{t} / /_{t}^{-1}(\mathscr{R} a)\left(X_{t}\right) d t+Q_{t} d n_{t} .
\end{aligned}
$$

The claim thus follows from Proposition 1.2.

COROllary 1.7. Fix $T>0$ and let $X_{t}(x)$ be a diffusion to $\mathscr{L}=\Delta+Z$, starting at $x$. Suppose that $a_{t}$ solves

$$
\left\{\begin{array}{l}
\frac{\partial}{\partial t} a_{t}=\left(\square-\mathscr{R}+\nabla_{Z}\right) a_{t} \quad \text { on }[0, T] \times M, \\
\left.a_{t}\right|_{t=0}=a \in \Gamma(E) .
\end{array}\right.
$$


Then

$$
N_{t}:=Q_{t} / /_{t}^{-1} a_{T-t}\left(X_{t}(x)\right), \quad 0 \leq t<T \wedge \zeta(x),
$$

is a local martingale, starting at $a_{T}(x)$. In particular, if $\zeta(x)=\infty$ and if equation (1.5) is a true martingale on $[0, T]$, we arrive at the formula

$$
a_{T}(x)=\mathbb{E}\left[Q_{T} / /_{T}^{-1} a\left(X_{T}(x)\right)\right], \quad a \in \Gamma(E) .
$$

Proof. Indeed, we have

$$
d N_{t} \stackrel{\mathrm{m}}{=} Q_{t} /_{t}^{-1} \underbrace{\left(\left(\square+\nabla_{Z}-\mathscr{R}\right) a_{T-t}+\frac{\partial}{\partial t} a_{T-t}\right)}_{=0}\left(X_{t}\right) d t=0
$$

as required.

REMARK 1.8. Note that

$$
\frac{d}{d t} Q_{t}=-Q_{t} \mathscr{R}_{/ / t} \quad \text { with } Q_{0}=\operatorname{id}_{E_{x}}
$$

implies the obvious estimate

$$
\left\|Q_{t}\right\|_{\text {op }} \leq \exp \left(-\int_{0}^{t} \underline{\mathscr{R}}\left(X_{s}(x)\right) d s\right)
$$

where $\underline{\mathscr{R}}(x)=\inf \left\{\left\langle\mathscr{R}_{x} v, w\right\rangle: v, w \in E_{x},\|v\| \leq 1\right.$ and $\left.\|w\| \leq 1\right\}$.

1.2. Commutation formulae. In the sequel, we consider the special case $E=$ $T^{*} M$. Thus $\Gamma(E)$ is the space of differential 1 -forms on $M$. The results of this section apply to vector fields as well, by identifying vector fields $V \in \Gamma(T M)$ and 1 -forms $\alpha \in \Gamma\left(T^{*} M\right)$ via the metric

$$
V \longleftrightarrow V^{b}, \quad \alpha \longleftrightarrow \alpha^{\#}
$$

Let $Z \in \Gamma(T M)$ be a vector field on $M$. Then the divergence of $Z$, denoted by $\operatorname{div} Z \in \mathscr{C}^{\infty}(M)$, is defined by $\operatorname{div} Z:=\operatorname{trace}\left(v \mapsto \nabla_{v} Z\right)$. Therefore,

$$
(\operatorname{div} Z)(x)=\sum_{i=1}^{n}\left\langle\nabla_{v_{i}} X, v_{i}\right\rangle
$$

for any orthonormal basis $\left\{v_{i}\right\}_{i=1}^{n}$ for $T_{x} M$. For compactly supported $f$, we have

$$
\langle Z, \nabla f\rangle_{L^{2}(T M)}=-\langle\operatorname{div} Z, f\rangle_{L^{2}(M)} .
$$

The adjoint $Z^{*}$ of $Z$ is given by the relation

$$
Z^{*} f=-Z f-(\operatorname{div} Z) f, \quad f \in C^{\infty}(M) .
$$


If either $f$ or $h$ is compactly supported, this implies

$$
\langle Z f, h\rangle_{L^{2}(M)}=\left\langle f, Z^{*} h\right\rangle_{L^{2}(M)} .
$$

Similarly, for $\alpha \in \Gamma\left(T^{*} M\right)$, we let

$$
(\operatorname{div} \alpha)(x)=\operatorname{trace}\left(T_{x} M \stackrel{\nabla \alpha}{\longrightarrow} T_{x}^{*} M \stackrel{\#}{\longrightarrow} T_{x} M\right) .
$$

Thus $\operatorname{div} Y=\operatorname{div} Y^{\mathrm{b}}$ and $\operatorname{div} \alpha=\operatorname{div} \alpha^{\#}$. That is, if $\delta=d^{*}$ denotes the usual codifferential then $\operatorname{div} \alpha=-\delta \alpha$. Finally, we define

$$
\operatorname{Ric}_{Z}(X, Y):=\operatorname{Ric}(X, Y)-\left\langle\nabla_{X} Z, Y\right\rangle, \quad X, Y \in \Gamma(T M) .
$$

NotATION 1.9. For the sake of convenience, we read bilinear forms on $M$, such as $\operatorname{Ric}_{Z}$, likewise as sections of $\operatorname{End}\left(T^{*} M\right)$ or $\operatorname{End}(T M)$, for example,

$$
\begin{array}{ll}
\operatorname{Ric}_{Z}(\alpha):=\operatorname{Ric}_{Z}\left(\cdot, \alpha^{\sharp}\right), & \alpha \in T^{*} M, \\
\operatorname{Ric}_{Z}(v):=\operatorname{Ric}_{Z}(v, \cdot)^{\sharp}, & v \in T M .
\end{array}
$$

If there is no risk of confusion, we do not distinguish in notation. In particular, depending on the context, $\left(\operatorname{Ric}_{Z}\right)_{/ / t}$ may be a random section of $\operatorname{End}\left(T^{*} M\right)$ or of $\operatorname{End}(T M)$.

LEMMA 1.10 (Commutation rules). Let $Z \in \Gamma(T M)$.

(1) For the differential d, we have

$$
d(\Delta+Z)=\left(\square-\operatorname{Ric}_{Z}+\nabla_{Z}\right) d ;
$$

(2) for the codifferential $d^{*}=-$ div, we have

$$
\left(\Delta+Z^{*}\right) d^{*}=d^{*}\left(\square-\operatorname{Ric}_{Z}^{*}+\nabla_{Z}^{*}\right),
$$

where the formal adjoint of $\nabla_{Z}$ (acting on 1 -forms) is $\nabla_{Z}^{*} \alpha=-\nabla_{Z} \alpha-(\operatorname{div} Z) \alpha$.

PROOF. Indeed, for any smooth function $f$ we have

$$
\begin{aligned}
d(\Delta+Z) f & =d\left(-d^{*} d f+(d f) Z\right) \\
& =\Delta^{(1)} d f+\nabla_{Z} d f+\langle\nabla \cdot Z, \nabla f\rangle \\
& =\left(\square+\nabla_{Z}\right)(d f)-\operatorname{Ric}_{Z}(\cdot, \nabla f) \\
& =\left(\square-\operatorname{Ric}_{Z}+\nabla_{Z}\right)(d f) .
\end{aligned}
$$

The formula in (2) is then just dual to (1). 
1.3. A formula for the differential. Now, let $X_{t}(x)$ be a diffusion to $\Delta+Z$ on $M$, starting at $X_{0}(x)=x, U_{t}$ a horizontal lift of $X_{t}(x)$ to $\mathrm{O}(M)$ and $B=$ $U_{0} \int_{U} \vartheta$ the martingale part of the antidevelopment of $X_{t}(x)$ to $T_{x} M$. Let $Q_{t}$ be the $\operatorname{Aut}\left(T_{x}^{*} M\right)$-valued process defined by

$$
\frac{d}{d t} Q_{t}=-Q_{t}\left(\operatorname{Ric}_{Z}\right) / / t
$$

with $Q_{0}=\operatorname{id}_{T_{x}^{*} M}$, let

$$
P_{t} f(x)=\mathbb{E}\left[\mathbb{1}_{\{t<\zeta(x)\}} f\left(X_{t}(x)\right)\right]
$$

be the minimal semigroup generated by $\Delta+Z$ on $M$, acting on bounded measurable functions $f$.

Fix $T>0$ and let $\ell_{t}$ be an adapted process with paths in the Cameron-Martin space $L^{1,2}\left([0, T] ; T_{x} M\right)$. By Corollary 1.7 ,

$$
N_{t}:=Q_{t} / /_{t}^{-1}\left(d P_{T-t} f\right), \quad t<T \wedge \zeta(x),
$$

is local martingale. Therefore,

$$
N_{t}\left(\ell_{t}\right)-\int_{0}^{t} Q_{s} / /_{s}^{-1}\left(d P_{T-s} f\right)\left(\dot{\ell}_{s}\right) d s
$$

is a local martingale. By integration by parts,

$$
\int_{0}^{t} Q_{s} / /_{s}^{-1}\left(d P_{T-s} f\right)\left(\dot{\ell}_{s}\right) d s-\frac{1}{2}\left(P_{T-t} f\right)\left(X_{t}(x)\right) \int_{0}^{t}\left\langle Q_{s}^{\operatorname{tr}}\left(\dot{\ell}_{s}\right), d B_{s}\right\rangle
$$

is also a local martingale and, therefore,

$$
Q_{t} / /_{t}^{-1}\left(d P_{T-t} f\right)\left(\ell_{t}\right)-\frac{1}{2}\left(P_{T-t} f\right)\left(X_{t}(x)\right) \int_{0}^{t}\left\langle Q_{s}^{\mathrm{tr}} \dot{e}_{s}, d B_{s}\right\rangle
$$

is a local martingale, starting at $\left(d P_{T} f\right)\left(\ell_{0}\right)$. Choosing $\ell_{t}$ so that (1.7) is a true martingale on $[0, T]$ with $\ell_{0}=v$ and $\ell_{T}=0$, we obtain the formula

$$
\left(d P_{T} f\right)(v)=-\frac{1}{2} \mathbb{E}\left[\mathbb{1}_{\{T<\zeta(x)\}} f\left(X_{T}(x)\right) \int_{0}^{T}\left\langle Q_{s}^{\mathrm{tr}} \dot{\ell}_{s}, d B_{s}\right\rangle\right] .
$$

For further details, see $[15,16]$. Denoting by $p_{t}(x, y)$ the smooth heat kernel associated to $\Delta+Z$, since formula (1.8) holds for all smooth functions $f$ of compact support, it implies Bismut's formula

$$
\left(d \log p_{T}(\cdot, y)\right)_{x}(v)=-\frac{1}{2} \mathbb{E}\left[\int_{0}^{\tau \wedge T}\left\langle Q_{s}^{\mathrm{tr}} \dot{\ell}_{s}, d B_{s}\right\rangle \mid X_{T}(x)=y\right] .
$$

The argument leading to formula (1.8) is based on the fact that the local martingale (1.7) is a true martingale. Since the condition on $\ell_{t}$ is imposed on the left endpoint, this can always be achieved, by taking $\ell_{s}=0$ for $s \geq \tau \wedge T$ where $\tau$ is the first exit time of some relatively compact neighborhood of $x$. No bounds on the 
geometry are needed; also explosion in finite times of the underlying diffusion can be allowed. For the problem of constructing appropriate finite energy processes $\ell_{s}$ with the property $\ell_{s}=0$ for $s \geq \tau \wedge T$, see [16], respectively, [17], Lemma 4.3.

Imposing in (1.7) however the conditions $\ell_{0}=0$ and $\ell_{T}=v$ would lead to a formula for

$$
\mathbb{E}\left[Q_{T} / /_{T}^{-1}(d f)_{X_{T}(x)}(v)\right]
$$

not involving derivatives of $f$, which clearly requires strong assumptions. If the local martingale (1.6) is a true martingale, we get the formula

$$
\left(d P_{T} f\right)_{x}(v)=\mathbb{E}\left[Q_{T} / /_{T}^{-1}(d f)_{X_{T}(x)}(v)\right] .
$$

For such a formula to hold, obviously $X_{t}(x)$ needs to be nonexplosive.

1.4. A formula for the codifferential. Recall that, according to Lemma 1.10, we have

$$
(\Delta+Z+\operatorname{div} Z) \operatorname{div}=\operatorname{div}\left(\square+\nabla_{Z}-\operatorname{Ric}_{-Z}^{*}+\operatorname{div} Z\right) .
$$

For a bounded 1-form $\alpha$ suppose $\alpha_{t}$ satisfies

$$
\frac{d}{d t} \alpha_{t}=\left(\square+\nabla_{Z}-\operatorname{Ric}_{-Z}^{*}+\operatorname{div} Z\right) \alpha_{t}
$$

with $\alpha_{0}=\alpha$, where $\operatorname{div} Z$ acts fibrewise as a multiplication operator, and that $\Theta_{t}$ is the $\operatorname{Aut}\left(T_{x} M\right)$-valued process which solves

$$
\frac{d}{d t} \Theta_{t}=-\left(\operatorname{Ric}_{-Z}^{*}-\operatorname{div} Z\right)_{/ / t} \Theta_{t}
$$

with $\Theta_{0}=\mathrm{id}_{T_{x} M}$. Here, Ricc $_{-Z}^{*}$ is the adjoint to Ric $-Z$ acting as endomorphism of $T_{x} M$; see Notation 1.9 .

REMARK 1.11. We have $\Theta_{t}=Q_{t}^{\text {tr }}$ if we set $\mathscr{R}:=\operatorname{Ric}_{-Z}^{*}-\operatorname{div} Z \in$ $\operatorname{End}\left(T^{*} M\right)$ and define $Q_{t}$ via Definition 1.5.

Proposition 1.12. Fix $T>0$. Let $X_{t}(x)$ be a diffusion to $\Delta+Z$ on $M$, starting at $x$.

(i) Then

$$
\left(\operatorname{div} \alpha_{T-t}\right)\left(X_{t}(x)\right) \exp \left(\int_{0}^{t}(\operatorname{div} Z)\left(X_{s}(x)\right) d s\right)
$$

is a local martingale, starting at $\operatorname{div} \alpha_{T}$.

(ii) Suppose $h_{t}$ is an adapted process with paths in $L^{1,2}([0, T] ; \mathbb{R})$. Then

(1.11) $\operatorname{div} \alpha_{T-t} h_{t}-\frac{1}{2} \alpha_{T-t}\left(/ / \Theta_{t} \int_{0}^{t}\left(\dot{h}_{s}-(\operatorname{div} Z)\left(X_{s}(x)\right) h_{s}\right) \Theta_{s}^{-1} / / s_{s}^{-1} d B_{s}\right)$ is a local martingale, starting at $\operatorname{div} \alpha_{T} h_{0}$. 
PROOF. (i) Taking into account the commutation rule (1.9) and the evolution equation (1.10) of $\alpha_{t}$, we get

$$
\begin{aligned}
\partial_{t} \operatorname{div} \alpha_{t} & =\operatorname{div} \partial_{t} \alpha_{t} \\
& =\operatorname{div}\left(\square+\nabla_{Z}-\operatorname{Ric}_{-Z}^{*}+\operatorname{div} Z\right) \alpha_{t} \\
& =(\Delta+Z+\operatorname{div} Z) \operatorname{div} \alpha_{t} .
\end{aligned}
$$

The claim then follows from Itô's formula.

(ii) To verify the second item, set

$$
\mathbb{A}_{t}:=\exp \left(\int_{0}^{t}(\operatorname{div} Z)\left(X_{s}(x)\right) d s\right)
$$

and define $\ell_{t}:=\mathbb{A}_{t}^{-1} h_{t}$. Using the fact that $\alpha_{T-t}\left(/ /_{t} \Theta_{t}\right)$ is a local martingale, indeed

$$
d\left(\alpha_{T-t}\left(/ / \Theta_{t}\right)\right)=\sum_{i=1}^{n}\left(\nabla_{U_{t} e_{i}} \alpha_{T-t}\right)\left(/ / \Theta_{t} \Theta_{t}\right) d B_{t}^{i}
$$

we obtain

$$
\begin{aligned}
\left(\operatorname{div} \alpha_{T-t}\right)\left(X_{t}(x)\right) \mathbb{A}_{t} \dot{\ell}_{t} d t \\
=\sum_{i=1}^{n}\left(\nabla_{U_{t} e_{i}} \alpha_{T-t}\right)\left(U_{t} e_{i}\right) \mathbb{A}_{t} \dot{\ell}_{t} d t \\
=\sum_{i=1}^{n}\left(/ /{ }_{t}^{-1} \nabla_{U_{t} e_{i}} \alpha_{T-t}\right)\left(U_{0} e_{i}\right) \mathbb{A}_{t} \dot{\ell}_{t} d t \\
=\sum_{i=1}^{n}\left(\nabla_{U_{t} e_{i}} \alpha_{T-t}\right)\left(/ / \Theta_{t} \Theta_{t} \Theta_{t}^{-1} U_{0} e_{i}\right) \mathbb{A}_{t} \dot{\ell}_{t} d t \\
=\frac{1}{2}\left\langle\sum_{i=1}^{n}\left(\nabla_{U_{t} e_{i}} \alpha_{T-t}\right)\left(/ / \Theta_{t}\right) d B_{t}^{i}, \mathbb{A}_{t} \dot{\ell}_{t} \Theta_{t}^{-1} d B_{t}\right\rangle \\
\quad \underline{m} \frac{1}{2} d\left(\alpha_{T-t}\left(/ / \Theta_{t} \Theta_{0}^{t} \mathbb{A}_{s} \dot{\ell}_{s} \Theta_{s}^{-1} d B_{s}\right)\right),
\end{aligned}
$$

where $\stackrel{m}{=}$ denotes equality modulo the differential of a local martingale. By part (i),

$$
n_{t}:=\left(\operatorname{div} \alpha_{T-t}\right)\left(X_{t}(x)\right) \mathbb{A}_{t}
$$

is a local martingale and, therefore, so is

$$
n_{t} \ell_{t}-\int_{0}^{t} n_{s} d \ell_{s}
$$


Since

$$
\mathbb{A}_{t} \dot{\ell}_{t}=\dot{h}_{t}-(\operatorname{div} Z)\left(X_{t}(x)\right) h_{t}
$$

the result follows by substitution.

REMARK 1.13. (a) Let $D^{n}$ be an exhausting sequence of $M$ by relatively compact open domains. Following the discussion of [3], Appendix B, and [9], Section III.1, it is standard to show that there is a strongly continuous semigroup $P_{t}^{n}$ on compactly supported 1 -forms $\alpha$ on $D^{n}$ generated by $L:=\square+\nabla_{Z}-$ Ric $_{-Z}^{*}+\operatorname{div} Z$ with Dirichlet boundary conditions. In probabilistic terms, $\alpha_{t}^{n}(x):=\left(P_{t}^{n} \alpha\right)(x)$ is easily identified as

$$
\alpha_{t}^{n}(x)=\mathbb{E}\left[\mathbb{1}_{\left\{t<\tau^{n}(x)\right\}} \alpha\left(/ / \Theta_{t}\right)\right],
$$

where $\tau^{n}(x)$ is the first exit time of $X_{t}(x)$ from $D^{n}$, when started at $x \in D^{n}$. As $n \rightarrow \infty$, the semigroup $\alpha_{t}^{n}$ converges to

$$
\alpha_{t}(x)=\mathbb{E}\left[\mathbb{1}_{\{t<\zeta(x)\}} \alpha\left(/ /_{t} \Theta_{t}\right)\right] .
$$

In particular, $\alpha_{t}$ solves equation (1.10) on $M$.

(b) Formula (1.13) shows that $\alpha_{t}$ is bounded in case $\alpha$ is bounded. Choosing the process $h$ in (1.11) in such a way that $h_{0}=1$ but $h_{t}=0$ for $t \geq \tau \wedge T$ where $\tau$ is the first exit time of $X_{t}(x)$ of some relatively compact neighborhood of $x$, we arrive at the formula

$$
\begin{aligned}
\left(\operatorname{div} \alpha_{T}\right)(x)=- & \frac{1}{2} \mathbb{E}\left[\mathbb { 1 } _ { \{ T < \zeta ( x ) \} } \alpha \left(/ /_{T} \Theta_{T} \int_{0}^{T}\left(\dot{h}_{S}-(\operatorname{div} Z)\left(X_{S}(x)\right) h_{S}\right)\right.\right. \\
& \left.\left.\times \Theta_{s}^{-1} / /_{s}^{-1} d B_{S}\right)\right] .
\end{aligned}
$$

Note that the local formula (1.14) does not require assumptions, either on the geometry of $M$ or on the drift vector field $Z$. Indeed, with an appropriate choice of $h$ it is always possible to make (1.11) a true martingale.

LEMMA 1.14. Suppose $\operatorname{Ric}_{Z}$ is bounded below, that Ric $+(\nabla . Z)^{*}, \operatorname{div} Z$ and $\operatorname{div} \alpha$ are bounded with $h_{t}$ bounded and

$$
\left(\int_{0}^{T}\left|\dot{h}_{s}\right|^{2} d s\right)^{1 / 2} \in L^{1+\epsilon}
$$

for some $\epsilon>0$. Then the local martingale (1.11) is a true martingale.

ProOF. Since $\mathrm{Ric}_{Z}$ is bounded below, the process $X_{t}$ is nonexplosive, by [19], Corollary 2.1.2. In this case, we have $\alpha_{t}=\mathbb{E}\left[\alpha\left(/ / \Theta_{t}\right)\right]$. From equation (1.12), we see that

$$
u(t, x):=\left(\operatorname{div} \alpha_{t}\right)(x)
$$


solves the heat equation

$$
\partial_{t} u=(\Delta+Z+\operatorname{div} Z) u
$$

with initial condition $u(0, \cdot)=\operatorname{div} \alpha$. By means of equation (1.14), combined with the bound on $\operatorname{div} Z$ and the other assumptions, we see that $\operatorname{div} \alpha_{t}$ is a bounded solution to (1.15), which implies

$$
\operatorname{div} \alpha_{t}=\mathbb{E}\left[(\operatorname{div} \alpha)\left(X_{t}\right) \exp \left(\int_{0}^{t}(\operatorname{div} Z)\left(X_{S}\right) d s\right)\right]
$$

for all $t \geq 0$. Note that our assumptions control the norms of $\Theta_{t}$ and $\Theta_{t}^{-1}$. Combined with the assumptions on $h$ this proves that (1.11) is indeed a true martingale.

REMARK 1.15. Equation (1.16) shows that div commutes with the semigroup $P_{t}^{(1)} \alpha:=\alpha_{t}$ on 1 -forms:

$$
\operatorname{div} P_{t}^{(1)} \alpha=P_{t}^{\operatorname{div} Z}(\operatorname{div} \alpha)
$$

where

$$
P_{t}^{\rho} f:=\mathbb{E}\left[f\left(X_{t}\right) \exp \left(\int_{0}^{t} \rho\left(X_{S}\right) d s\right)\right]
$$

denotes the Feynman-Kac semigroup on functions to $\Delta+Z$ with scalar potential $\rho$.

Using the identification of differential forms and vector fields via the metric, we obtain the following result (which for compact $M$ with $Z=0$ corrects the sign in [3], Theorem 5.10).

THEOREM 1.16. Let $M$ be a Riemannian manifold and $Z$ a smooth vector field on $M$. Let $X=X(x)$ be a diffusion to $\Delta+Z$ on $M$, starting at $X_{0}(x)=x$, which is assumed to be nonexplosive. Let $T>0$ and $h$ be an adapted process with paths in $L^{1,2}([0, T] ; \mathbb{R})$ such that $h_{0}=0$ and $h_{T}=1$, and such that (1.11) is a true martingale. Then for all bounded smooth vector fields $V$ on $M$,

$$
\begin{aligned}
& \mathbb{E}\left[(\operatorname{div} V)\left(X_{T}(x)\right)\right] \\
& \quad=\frac{1}{2} \mathbb{E}\left[\left\langle V\left(X_{T}(x)\right), / /_{T} \Theta_{T} \int_{0}^{T}\left(\dot{h}_{t}-(\operatorname{div} Z)\left(X_{t}(x)\right) h_{t}\right) \Theta_{t}^{-1} d B_{t}\right\rangle\right],
\end{aligned}
$$

where $\Theta$ is the $\operatorname{Aut}\left(T_{x} M\right)$-valued process defined by the following pathwise differential equation:

$$
\frac{d}{d t} \Theta_{t}=-\operatorname{Ric}_{/ / t} \Theta_{t}-(\nabla . Z)_{/ / t}^{*} \Theta_{t}+(\operatorname{div} Z) \Theta_{t}
$$

with $\Theta_{0}=\mathrm{id}_{T_{x} M}$. 
COROLlaRY 1.17. Suppose $f$ is a bounded smooth function and that $V$ is a bounded smooth vector field with $\operatorname{div} V$ bounded. Then, under the assumptions of Theorem 1.16, by using the relation $\operatorname{div}(f V)=V f+f \operatorname{div} V$, we get

$$
\begin{aligned}
P_{T}(V(f))(x) & \\
=- & \mathbb{E}\left[f\left(X_{T}(x)\right)(\operatorname{div} V)\left(X_{T}(x)\right)\right] \\
+ & \frac{1}{2} \mathbb{E}\left[f ( X _ { T } ( x ) ) \left\langleV\left(X_{T}(x)\right),\right.\right. \\
& \left.\left.\quad /{ }_{T} \Theta_{T} \int_{0}^{T}\left(\dot{h}_{t}-(\operatorname{div} Z)\left(X_{t}(x)\right) h_{t}\right) \Theta_{t}^{-1} d B_{t}\right\rangle\right],
\end{aligned}
$$

where the right-hand side does not contain any derivatives of $f$.

COROLLARY 1.18. Under the assumptions of Theorem 1.16, we have

$\left(\nabla \log p_{T}(x, \cdot)\right)_{y}$

$$
=-\frac{1}{2} \mathbb{E}\left[/ / \Theta_{T} \int_{0}^{T}\left(\dot{h}_{t}-(\operatorname{div} Z)\left(X_{t}(x)\right) h_{t}\right) \Theta_{t}^{-1} d B_{t} \mid X_{T}(x)=y\right]
$$

with $\Theta$ given as above.

PROOF. By Theorem 1.16, for all smooth, compactly supported vector fields $V$ we have

$$
\begin{aligned}
& P_{T}(\operatorname{div} V)(x) \\
& =\frac{1}{2} \int_{M}\left\langle V, \mathbb{E}\left[/ /_{T} \Theta_{T} \int_{0}^{T}\left(\dot{h}_{t}-(\operatorname{div} Z)\left(X_{t}(x)\right) h_{t}\right)\right.\right. \\
& \left.\left.\quad \Theta_{t}^{-1} d B_{t} \mid X_{T}(x)=y\right]\right\rangle p_{T}(x, y) d y
\end{aligned}
$$

but on the other hand

$$
\begin{aligned}
P_{T}(\operatorname{div} V)(x) & =\int_{M}(\operatorname{div} V)(y) p_{T}(x, y) d y \\
& =-\int_{M}\left(d p_{T}(x, \cdot)\right)_{y} V(y) d y \\
& =-\int_{M}\left(d \log p_{T}(x, \cdot)\right)_{y} V(y) p_{T}(x, y) d y
\end{aligned}
$$

so the result follows. 
1.5. Shift-Harnack inequalities. Suppose $\operatorname{Ric}_{Z}$ is bounded below, that Ric + $(\nabla . Z)^{*}$ and $\operatorname{div} Z$ are bounded and that the following formula holds, for all $t>0$, all $f \in C_{b}^{1}(M)$ and all bounded vector fields $V$ with $\operatorname{div} V$ bounded (see Corollary 1.17):

$$
\begin{aligned}
P_{t}(V(f))(x)=- & \mathbb{E}\left[f\left(X_{t}(x)\right)(\operatorname{div} V)\left(X_{t}(x)\right)\right] \\
+ & \frac{1}{2} \mathbb{E}\left[f ( X _ { t } ( x ) ) \left\langleV\left(X_{t}(x)\right),\right.\right. \\
& \left.\left.\quad / / \Theta_{t} \int_{0}^{t}\left[\frac{1}{t}-(\operatorname{div} Z)\left(X_{r}(x)\right) \frac{r}{t}\right] \Theta_{r}^{-1} d B_{r}\right\rangle\right] .
\end{aligned}
$$

Fix $T>0$. Then, by Jensen's inquality (see [14], Lemma 6.45), there exist constants $c, C_{1}(T)>0$ such that

$$
\begin{aligned}
\left|P_{t}(V(f))\right| \leq & \delta\left(P_{t}(f \log f)-P_{t} f \log P_{t} f\right) \\
& +\underbrace{\left(|\operatorname{div} V|_{\infty}+\delta c+\frac{C_{1}(T)}{\delta t}|V|_{\infty}^{2}\right)}_{=: \alpha_{1}(\delta, t, V)} P_{t} f
\end{aligned}
$$

for all $\delta>0, t \in(0, T]$ and positive $f \in C_{b}^{1}(M)$. Alternatively, by the CauchySchwarz inequality, there exists $C_{2}(T)>0$ such that

$$
\left|P_{t}(V(f))\right|^{2} \leq \underbrace{\left(|\operatorname{div} V|_{\infty}+\frac{C_{2}(T)}{\sqrt{t}}|V|_{\infty}\right)^{2}}_{=: \alpha_{2}(t, V)} P_{t} f^{2}
$$

for all $t \in(0, T]$ and $f \in C_{b}^{1}(M)$. These estimates can be used to derive shiftHarnack inequalities, as shown by F.-Y. Wang for the case of a Markov operator on a Banach space (see [18], Proposition 2.3). In particular, suppose $\left\{F_{s}: s \in[0,1]\right\}$ is a $C^{1}$ family of diffeomorphisms of $M$ with $F_{0}=\operatorname{id}_{M}$. For each $s \in[0,1]$, define a vector field $V_{s}$ on $M$ by

$$
V_{s}:=\left(D F_{s}\right)^{-1} \dot{F}_{S}
$$

and assume $V_{s}$ and $\operatorname{div} V_{s}$ are uniformly bounded. Note $\frac{d}{d s}\left(f \circ F_{s}\right)=\nabla_{V_{s}}(f \circ$ $\left.F_{s}\right)$. Fixing $p \geq 1$ and setting $\beta(s)=1+(p-1) s$, as in the first part of [18], Proposition 2.3, we deduce from inequality (1.17) that

$$
\frac{d}{d s} \log \left(P_{t}\left(f^{\beta(s)} \circ F_{s}\right)\right)^{p / \beta(s)} \geq-\frac{p}{\beta(s)} \alpha_{1}\left(\frac{\beta^{\prime}(s)}{\beta(s)}, t, V_{s}\right)
$$

for all $s \in[0,1]$, which when integrated gives the shift-Harnack inequality

$$
\left(P_{t} f\right)^{p} \leq\left(P_{t}\left(f^{p} \circ F_{1}\right)\right) \exp \left(\int_{0}^{1} \frac{p}{\beta(s)} \alpha_{1}\left(\frac{\beta^{\prime}(s)}{\beta(s)}, t, V_{s}\right) d s\right)
$$


for each $t \in[0, T]$ and positive $f \in C_{b}^{1}(M)$. Alternatively, from inequality (1.18) and following the calculation in the second part of [18], Proposition 2.3, we deduce

$$
P_{t} f \leq P_{t}\left(f \circ F_{1}\right)+\left(\int_{0}^{1} \alpha_{2}\left(t, V_{s}\right) d s\right)^{1 / 2} \sqrt{P_{t} f^{2}}
$$

for each $t \in[0, T]$ and positive $f \in C_{b}^{1}(M)$. The shift $F_{1}$ could be given by the exponential of a well-behaved vector field; the shifts considered in [18] are of the form $x \mapsto x+v$, for some $v$ belonging to the Banach space.

2. Extrinsic formulae. Suppose now that $M$ is simply a smooth manifold of dimension $n$. Suppose $A_{0}$ is a smooth vector field and

$$
A: M \times \mathbb{R}^{m} \rightarrow T M, \quad(x, e) \mapsto A(x) e,
$$

a smooth bundle map over $M$. This means $A(\cdot) e$ is a vector field on $M$ for each $e \in \mathbb{R}^{m}$, and $A(x): \mathbb{R}^{m} \rightarrow T_{x} M$ is linear for each $x \in M$.

For an $\mathbb{R}^{m}$-valued Brownian motion $B_{t}$, sped up by 2 so that $d[B, B]_{t}=$ $2 \operatorname{id}_{\mathbb{R}^{m}} d t$, defined on a filtered probability space $\left(\Omega, \mathscr{F}, \mathbb{P} ;\left(\mathscr{F}_{t}\right)_{t \in \mathbb{R}_{+}}\right)$, satisfying the usual completeness conditions, consider the Stratonovich stochastic differential equation

$$
d X_{t}=A_{0}\left(X_{t}\right) d t+A\left(X_{t}\right) \circ d B_{t} .
$$

Given an orthonormal basis $\left\{e_{i}\right\}_{i=1}^{m}$ of $\mathbb{R}^{m}$ set $A_{i}(\cdot):=A(\cdot) e_{i}$ and $B_{t}^{i}:=\left\langle B_{t}, e_{i}\right\rangle$. Then the previous equation can be equivalently written

$$
d X_{t}=A_{0}\left(X_{t}\right) d t+\sum_{i=1}^{m} A_{i}\left(X_{t}\right) \circ d B_{t}^{i} .
$$

There is a partial flow $X_{t}(\cdot), \zeta(\cdot)$ associated to (2.1) (see [11] for details) such that for each $x \in M$ the process $X_{t}(x), 0 \leq t<\zeta(x)$ is the maximal strong solution to (2.1) with starting point $X_{0}(x)=x$, defined up to the explosion time $\zeta(x)$; moreover, using the notation $X_{t}(x, \omega)=X_{t}(x)(\omega)$ and $\zeta(x, \omega)=\zeta(x)(\omega)$, if

$$
M_{t}(\omega)=\{x \in M: t<\zeta(x, \omega)\}
$$

then there exists $\Omega_{0} \subset \Omega$ of full measure such that for all $\omega \in \Omega_{0}$ :

(i) $M_{t}(\omega)$ is open in $M$ for each $t \geq 0$, that is, $\zeta(\cdot, \omega)$ is lower semicontinuous on $M$;

(ii) $X_{t}(\cdot, \omega): M_{t}(\omega) \rightarrow M$ is a diffeomorphism onto an open subset of $M$;

(iii) the map $s \mapsto X_{s}(\cdot, \omega)$ is continuous from $[0, t]$ into $C^{\infty}\left(M_{t}(\omega), M\right)$ with its $C^{\infty}$-topology, for each $t>0$.

The solution processes $X=X(x)$ to (2.1) are diffusions on $M$ with generator

$$
\mathscr{L}:=A_{0}+\sum_{i=1}^{m} A_{i}^{2} .
$$


We will assume that the equation is nondegenerate, which is to say that $A(x)$ : $\mathbb{R}^{m} \rightarrow T_{x} M$ is surjective for all $x \in M$. Then $A$ induces a Riemannian metric on $M$, the quotient metric, with respect to which

$$
A(x)^{*}=\left(\left.A(x)\right|_{\text {ker } A(x)^{\perp}}\right)^{-1}
$$

and whose inner product $\langle\cdot, \cdot\rangle$ on a tangent space $T_{x} M$ is given by

$$
\langle v, u\rangle=\left\langle A(x)^{*} v, A(x)^{*} u\right\rangle_{\mathbb{R}^{m}} .
$$

\subsection{A formula for the differential. Denote by}

$$
P_{t} f(x):=\mathbb{E}\left[\mathbb{1}_{\{t<\zeta(x)\}} f\left(X_{t}(x)\right)\right]
$$

the minimal semigroup associated to equation (2.1), acting on bounded measurable functions $f$. In terms of any linear connection $\tilde{\nabla}$ on $T M$ with adjoint $\tilde{\nabla}^{\prime}$ [see (2.5) below], a solution $T X_{t}(x)$ to the derivative equation

$$
d^{\tilde{\nabla}^{\prime}} T X_{t}(x)=\tilde{\nabla}_{T X_{t}(x)} A_{0} d t+\sum_{i=1}^{m} \tilde{\nabla}_{T X_{t}(x)} A_{i} \circ d B_{t}^{i}
$$

with $T X_{0}(x)=\operatorname{id}_{T_{x} M}$ is the derivative (in probability) at $x$ of the solution flow to (2.1). Our objective will be to find a formula for $P_{T}(V(f))$ in terms of $T X_{t}$. Before doing so, let us briefly derive the corresponding formula for $\left(d P_{T}\right)(v)$. As in Section 1.3, let $\ell_{t}$ be an adapted process with paths in $L^{1,2}\left([0, T] ; T_{x} M\right)$. By Itô's formula and the Weitzenböck formula (see [4], Theorem 2.4.2), it follows, according to the procedure of Section 1.3, that

$$
\left(d P_{T-t} f\right)\left(T X_{t}(x) \ell_{t}\right)-\frac{1}{2}\left(P_{T-t} f\right)\left(X_{t}(x)\right) \int_{0}^{t}\left\langle T X_{s}(x) \dot{\ell}_{s}, A\left(X_{s}(x)\right) d B_{s}\right\rangle
$$

is a local martingale, starting at $\left(d P_{T} f\right)\left(\ell_{0}\right)$. Choosing $\ell_{t}$ so that (2.2) is a true martingale with $\ell_{0}=v$ and $\ell_{T}=0$, we obtain the formula

$$
\left(d P_{T} f\right)(v)=-\frac{1}{2} \mathbb{E}\left[\mathbb{1}_{\{T<\zeta(x)\}} f\left(X_{T}(x)\right) \int_{0}^{T}\left\langle T X_{s}(x) \dot{\ell}_{s}, A\left(X_{S}(x)\right) d B_{s}\right\rangle\right] .
$$

This formula is well known; it is the one given by [15], Theorem 2.4. Formula (1.8) can be obtained from it by filtering. Furthermore, it as always possible to choose such $\ell_{t}$, as in Section 1.3. Now denote by $p_{t}(x, y)$ the smooth heat kernel associated to (2.1) such that

$$
P_{t} f(x)=\int_{M} f(y) p_{t}(x, y) \operatorname{vol}(d y)
$$

where $\operatorname{vol}(d y)$ denotes integration with respect to the induced Riemannian volume measure. Since formula (2.3) holds for all smooth functions $f$ of compact support, we deduce from it the Bismut formula

$$
\left(d \log p_{T}(\cdot, y)\right)_{x}(v)=-\frac{1}{2} \mathbb{E}\left[\int_{0}^{\tau \wedge T}\left\langle T X_{s}(x) \dot{\ell}_{s}, A\left(X_{S}(x)\right) d B_{S}\right\rangle \mid X_{T}(x)=y\right],
$$


the original version of which was given in [1] for compact manifolds. The version stated here is [15], Corollary 2.5, the nonlocal version having been earlier given in [7].

2.2. Induced linear connections. There are a number of linear connections naturally associated to the map $A$. First, there is the Levi-Civita connection $\nabla$ for the induced metric. Second, there is the Le Jan-Watanabe connection, which is given by the push forward under $A$ of the flat connection on $\mathbb{R}^{m}$. Its covariant derivative $\breve{\nabla}$ is defined by

$$
\breve{\nabla}_{v} U=A(x) d\left(A(\cdot)^{*} U(\cdot)\right)_{x}(v)
$$

for a vector field $U$ and $v \in T_{x} M$. Like the Levi-Civita connection, it is adapted to the induced metric. In fact, all metric connections on $T M$ arise in this way. In addition to the properties of $\breve{\nabla}$ summarized below, further details of it can be found in [4-6]. It has the property that if $e \in \operatorname{ker} A(x)^{\perp}$ then $\breve{\nabla}_{v} A_{e}=0$ for all $v \in T_{x} M$, where by $A_{e}$ we mean the section $x \mapsto A(x) e$. It therefore satisfies the Le Jan-Watanabe property

$$
\sum_{i=1}^{m} \breve{\nabla}_{A_{i}} A_{i}=0 .
$$

To any linear connection $\tilde{\nabla}$ on $T M$, one can associate an adjoint connection $\tilde{\nabla}^{\prime}$ by

$$
\tilde{\nabla}_{v}^{\prime} U=\tilde{\nabla}_{v} U-\tilde{T}(v, U)
$$

for $v$ a vector and $U$ a smooth vector field, where $\tilde{T}$ denotes the torsion tensor of $\tilde{\nabla}$. The adjoint of the Le Jan-Watanabe connection will be denoted by $\hat{\nabla}$. It therefore satisfies

$$
\hat{\nabla}_{v} U=\breve{\nabla}_{v} U-\breve{T}(v, U)
$$

or equivalently $\breve{\nabla}_{v} U=\hat{\nabla}_{v} U-\hat{T}(v, U)$, where $\breve{T}$ and $\hat{T}$ denote the torsion tensors of $\breve{\nabla}$ and $\hat{\nabla}$, respectively; these antisymmetric tensors satisfy $\breve{T}=-\hat{T}$. By [4], Proposition 2.2.3, the torsion can be written in terms of $A$ by

$$
\breve{T}(v, u)_{x}=A(x)\left(d A^{*}\right)_{x}(v, u),
$$

where $d A^{*}$ denotes the exterior derivative of the $\mathbb{R}^{m}$-valued 1-form $A^{*}: T M \rightarrow$ $\mathbb{R}^{m}$. The adjoint connection can therefore be written in terms of $A$ by

$$
\hat{\nabla}_{v} U=A(x)\left(d\left(A^{*}(\cdot) U(\cdot)\right)_{x}(v)-\left(d A^{*}\right)_{x}(v, U)\right) .
$$

Besides torsion, we will also encounter several expressions involving curvature, including

$$
\breve{\mathrm{Ric}}:=\sum_{i=1}^{m} \breve{R}\left(\cdot, A_{i}\right) A_{i},
$$


where $\breve{R}$ denotes the curvature tensor of $\breve{\nabla}$. In particular, [4], Lemma 2.4.3, states for a smooth 1 -form $\phi$ that

$$
\sum_{i=1}^{m} L_{A_{i}} L_{A_{i}} \phi=\operatorname{trace} \hat{\nabla}^{2} \phi-\phi(\widetilde{\mathrm{Ric}}),
$$

where $L$ denotes Lie differentiation.

2.3. Induced differential operators. With respect to the metric induced by $A$, we set $\delta:=d^{*}$. For a 1 -form $\phi$, the codifferential $\delta$ satisfies

$$
\delta \phi=-\sum_{i=1}^{m}\left(\nabla_{A_{i}} \phi\right)\left(A_{i}\right)
$$

but this relation does not hold with $\nabla$ replaced by $\hat{\nabla}$. Nonetheless, for the divergence of a smooth vector field $U$ we do have

$$
\operatorname{div} U=\sum_{i=1}^{m}\left\langle\nabla_{A_{i}} U, A_{i}\right\rangle=\sum_{i=1}^{m}\left\langle\hat{\nabla}_{A_{i}} U, A_{i}\right\rangle=\operatorname{trace} \hat{\nabla} U
$$

by the adaptedness of $\breve{\nabla}$.

LEMMA 2.1. For any smooth vector field $U, 1$-form $\phi$ and linear connection $\tilde{\nabla}$ with adjoint $\tilde{\nabla}^{\prime} U=\tilde{\nabla} \cdot U-\tilde{T}(\cdot, U)$ we have

$$
(U+\operatorname{div} U) \delta \phi=-\delta\left(\tilde{\nabla}_{U}^{*}+\left(\tilde{\nabla}^{\prime} U\right)^{*}\right) \phi .
$$

PROOF. As a linear connection, $\tilde{\nabla}$ satisfies

$$
L_{U} \phi=\tilde{\nabla}_{U} \phi+\phi\left(\tilde{\nabla}^{\prime} U\right) .
$$

Since $d$ commutes with Lie differentiation, we thus have

$$
\begin{aligned}
d U f & =L_{U} d f \\
& =\tilde{\nabla}_{U} d f+d f\left(\tilde{\nabla}^{\prime} U\right) \\
& =\tilde{\nabla}_{U} d f+\left(\tilde{\nabla}^{\prime} U\right) d f .
\end{aligned}
$$

By duality, this implies

$$
U^{*} \delta \phi=\delta\left(\tilde{\nabla}_{U}^{*}+\left(\tilde{\nabla}^{\prime} U\right)^{*}\right) \phi
$$

and, therefore,

$$
(U+\operatorname{div} U) \delta \phi=-\delta\left(\tilde{\nabla}_{U}^{*}+\left(\tilde{\nabla}^{\prime} U\right)^{*}\right) \phi
$$

since $U^{*}=-U-\operatorname{div} U$. 
With respect to the induced metric, the formal adjoint $\nabla_{U}^{*}$ of the differential operator $\nabla_{U}$ acting on 1 -forms is given by

$$
\nabla_{U}^{*}=-\nabla_{U}-\operatorname{div} U \text {. }
$$

More generally, we have the following lemma.

LEMMA 2.2. For any smooth vector field $U$ and metric connection $\tilde{\nabla}^{\prime}$ with adjoint $\tilde{\nabla}$, we have

$$
\tilde{\nabla}_{U}^{*}=-\tilde{\nabla}_{U}-\operatorname{div} U-\tilde{T}(U, \cdot)-\tilde{T}(U, \cdot)^{*} .
$$

PROOF. Denoting by $\mu_{g}$ the Riemannian volume density, the divergence of a vector field $U$ satisfies $L_{U} \mu_{g}=(\operatorname{div} U) \mu_{g}$ and thus for compactly supported 1 -forms $\phi, \psi$ we have

$$
\begin{aligned}
L_{U}\left(\langle\phi, \psi\rangle \mu_{g}\right)= & \left\langle\tilde{\nabla}_{U}^{\prime} \phi, \psi\right\rangle \mu_{g}+\left\langle\phi, \tilde{\nabla}_{U}^{\prime} \psi\right\rangle \mu_{g}+(\operatorname{div} U)\langle\phi, \psi\rangle \mu_{g} \\
= & \left\langle\tilde{\nabla}_{U} \phi, \psi\right\rangle \mu_{g}+\left\langle\phi, \tilde{\nabla}_{U} \psi\right\rangle \mu_{g}+\langle\phi(\tilde{T}(U, \cdot)), \psi\rangle \mu_{g} \\
& +\langle\phi, \psi(\tilde{T}(U, \cdot))\rangle \mu_{g}+(\operatorname{div} U)\langle\phi, \psi\rangle \mu_{g}
\end{aligned}
$$

from which the result follows, since $\int_{M} L_{U}\left(\langle\phi, \psi\rangle \mu_{g}\right)=0$, by Stokes' theorem.

The map $A$ also induces a differential operator $\hat{\delta}$, mapping 1-forms to functions by

$$
\hat{\delta} \phi:=-\sum_{i=1}^{m} \iota_{A_{i}} L_{A_{i}} \phi
$$

Since $L_{A_{i}} \phi=\iota_{A_{i}} d \phi+d\left(\iota_{A_{i}} \phi\right)$, the generator $\mathscr{L}$ can be expressed in terms of $\hat{\delta}$ by

$$
\mathscr{L}=L_{A_{0}}-(\hat{\delta} d+d \hat{\delta}) .
$$

Clearly, $\hat{\delta}^{2}=0$, so to find an analogue of the second commutation rule in Lemma 1.10 for $\hat{\delta}$ and $\mathscr{L}$ it suffices to calculate the Lie derivative of $\hat{\delta}$ in the direction $A_{0}$. This is the main objective of the remainder of this section. Note that $\hat{\delta}$ need not agree with the codifferential $\delta$. For any smooth vector field $U$ and linear connection $\tilde{\nabla}$ with adjoint $\tilde{\nabla}^{\prime}$, we have

$$
L_{U} \phi=\left(\tilde{\nabla}_{U} \phi\right)+\phi\left(\tilde{\nabla}^{\prime} U\right)
$$

and, therefore,

$$
\hat{\delta} \phi=-\sum_{i=1}^{m}\left(\hat{\nabla}_{A_{i}} \phi\right)\left(A_{i}\right)-\sum_{i=1}^{m} \phi\left(\breve{\nabla}_{A_{i}} A_{i}\right)=-\sum_{i=1}^{m}\left(\hat{\nabla}_{A_{i}} \phi\right)\left(A_{i}\right)
$$


or alternatively

$$
\hat{\delta} \phi=-\sum_{i=1}^{m}\left(\breve{\nabla}_{A_{i}} \phi\right)\left(A_{i}\right)-\sum_{i=1}^{m} \phi\left(\hat{\nabla}_{A_{i}} A_{i}\right)=-\sum_{i=1}^{m}\left(\breve{\nabla}_{A_{i}} \phi\right)\left(A_{i}\right)
$$

by the Le Jan-Watanabe property and the fact that $\breve{T}\left(A_{i}, A_{i}\right)=0$. Applying (2.11) to the Levi-Civita connection gives

$$
\hat{\delta} \phi=-\sum_{i=1}^{m}\left(\nabla_{A_{i}} \phi\right)\left(A_{i}\right)-\sum_{i=1}^{m} \phi\left(\nabla_{A_{i}} A_{i}\right)
$$

and so by (2.8) we have

$$
\hat{\delta} \phi=\delta \phi-\phi\left(\sum_{i=1}^{m} \nabla_{A_{i}} A_{i}\right)
$$

which expresses the difference of the operators $\delta$ and $\hat{\delta}$.

LEMMA 2.3. For any smooth vector field $U$ and 1-form $\phi$, we have

$$
\begin{aligned}
(U+ & \operatorname{trace} \hat{\nabla} U) \hat{\delta} \phi \\
& =\hat{\delta}\left(\hat{\nabla}_{U}-(\breve{\nabla} U)^{*}-\breve{T}(U, \cdot)-\breve{T}(U, \cdot)^{*}+\operatorname{trace} \hat{\nabla} U\right) \phi+\phi\left(U^{A}\right),
\end{aligned}
$$

where the vector field $U^{A}$ is defined by

$$
U^{A}:=-\sum_{i=1}^{m}\left((\hat{\nabla} U)^{*}+\hat{\nabla} U\right)\left(\nabla_{A_{i}} A_{i}\right)-\sum_{i=1}^{m}\left[U, \nabla_{A_{i}} A_{i}\right] .
$$

ProOF. By Lemmas 2.1 and 2.2, we have

$$
(U+\operatorname{div} U) \delta \phi=\delta\left(\hat{\nabla}_{U}+\operatorname{div} U+\hat{T}(U, \cdot)+\hat{T}(U, \cdot)^{*}-(\breve{\nabla} U)^{*}\right) \phi .
$$

By (2.14), we have

$$
\begin{aligned}
(U+\operatorname{div} U) \delta \phi= & (U+\operatorname{div} U) \hat{\delta} \phi+(\operatorname{div} U) \phi\left(\nabla_{A_{i}} A_{i}\right) \\
& +\left(\hat{\nabla}_{U} \phi\right)\left(\nabla_{A_{i}} A_{i}\right)+\phi\left(\hat{\nabla}_{U} \nabla_{A_{i}} A_{i}\right)
\end{aligned}
$$

and

$$
\begin{aligned}
\delta\left(\hat{\nabla}_{U}+\right. & \left.\operatorname{div} U+\hat{T}(U, \cdot)+\hat{T}(U, \cdot)^{*}-(\breve{\nabla} U)^{*}\right) \phi \\
= & \hat{\delta}\left(\hat{\nabla}_{U}+\operatorname{div} U-\breve{T}(U, \cdot)-\breve{T}(U, \cdot)^{*}-(\breve{\nabla} U)^{*}\right) \phi+\left(\hat{\nabla}_{U} \phi\right)\left(\nabla_{A_{i}} A_{i}\right) \\
& +\left(\left(\operatorname{div} U-\breve{T}(U, \cdot)-\breve{T}(U, \cdot)^{*}-(\breve{\nabla} U)^{*}\right) \phi\right)\left(\nabla_{A_{i}} A_{i}\right) .
\end{aligned}
$$

Rearranging, the result follows by equation (2.9). 
Note that the vector field $A_{0}^{A}$ appears to depend on the Levi-Civita connection via the sum of the vector fields $\nabla_{A_{i}} A_{i}$. It is clear that all other objects appearing in the definition of $A_{0}^{A}$ can be calculated explicitly in terms of $A$ and $A_{0}$, by formula (2.4). The following lemma, combined with formula (2.6), shows that the sum of the vector fields $\nabla_{A_{i}} A_{i}$ can also be expressed directly in terms of $A$.

LEMMA 2.4. We have

$$
\sum_{i=1}^{m} \nabla_{A_{i}} A_{i}=-\sum_{i=1}^{m} \breve{T}\left(\cdot, A_{i}\right)^{*}\left(A_{i}\right)
$$

where $\breve{T}$ denotes the torsion of the Le Jan-Watanabe connection.

PROOF. Suppressing the summation over $i$, the Le Jan-Watanabe property implies

$$
\nabla_{A_{i}} A_{i}=\breve{\nabla}_{A_{i}} A_{i}-\breve{K}\left(A_{i}, A_{i}\right)=-\breve{K}\left(A_{i}, A_{i}\right),
$$

where $\breve{K}$ denotes the contorsion tensor of $\breve{\nabla}$. The contorsion tensor measures the extent to which a metric connection fails to be the Levi-Civita connection, vanishing if the connection is torsion-free. It is discussed in [10] and [12]. The components of $\breve{K}$ satisfy $\breve{K}^{i}{ }_{j j}=\breve{T}_{j}{ }^{i}{ }_{j}$, which is to say

$$
\breve{K}\left(A_{i}, A_{i}\right)=\left(\breve{T}\left(\cdot, A_{i}\right)^{b}\right)\left(A_{i}\right)^{\sharp},
$$

where $b$ and $\sharp$ are the musical isomorphisms associated to the induced metric. This implies

$$
\left\langle\breve{K}\left(A_{i}, A_{i}\right), U\right\rangle=\left\langle\breve{T}\left(U, A_{i}\right), A_{i}\right\rangle
$$

for all smooth vector fields $U$ and, therefore,

$$
\breve{K}\left(A_{i}, A_{i}\right)=\breve{T}\left(\cdot, A_{i}\right)^{*}\left(A_{i}\right)
$$

as required.

Consequently,

$$
A_{0}^{A}=\sum_{i=1}^{m}\left(\hat{\nabla} A_{0}+\left(\hat{\nabla} A_{0}\right)^{*}\right)\left(\breve{T}\left(\cdot, A_{i}\right)^{*}\left(A_{i}\right)\right)+\left[A_{0}, \breve{T}\left(\cdot, A_{i}\right)^{*}\left(A_{i}\right)\right] .
$$

2.4. Commutation formula. We have, in summary, the following commutation rule, extending formula (1.9).

PROPOSITION 2.5. For any smooth 1-form $\phi$, we have

$$
\begin{aligned}
(\mathscr{L}+ & \left.\operatorname{trace} \hat{\nabla} A_{0}\right) \hat{\delta} \phi \\
& =\hat{\delta}\left(\operatorname{trace} \hat{\nabla}^{2}+\hat{\nabla}_{A_{0}}-\mathrm{Ric}_{A_{0}}-\hat{\nabla} A_{0}-\left(\hat{\nabla} A_{0}\right)^{*}+\operatorname{trace} \hat{\nabla} A_{0}\right) \phi+\phi\left(A_{0}^{A}\right),
\end{aligned}
$$

where the vector field $A_{0}^{A}$ is given by (2.15) and $\breve{\mathrm{Ric}_{A_{0}}}:=\mathrm{Ric}-\breve{\nabla} A_{0}$. 
Proof. The claim follows from Lemmas 2.3 and 2.4 and the relations (2.7) and (2.10).

Finally, note that for a smooth function $f$, the codifferential $\delta$ satisfies

$$
\langle d f, \phi\rangle=f \delta(\phi)-\delta(f \phi) .
$$

We will need an analogous formula for $\hat{\delta}$, as given by the following lemma.

LEMMA 2.6. For any smooth function $f$, we have

$$
\langle d f, \phi\rangle=f \hat{\delta}(\phi)-\hat{\delta}(f \phi) .
$$

PROOF. Suppressing notationally the summation over $i$, we have

$$
\begin{aligned}
\hat{\delta}(f \phi) & =-\iota_{A_{i}} L_{A_{i}}(f \phi) \\
& =-\iota_{A_{i}}\left(\iota_{A_{i}} d(f \phi)+d\left(\iota_{A_{i}} f \phi\right)\right) \\
& =-\iota_{A_{i}}\left(\iota_{A_{i}}(d f \wedge \phi+f d \phi)+\phi\left(A_{i}\right) d f+f d\left(\phi\left(A_{i}\right)\right)\right) \\
& =-\iota_{A_{i}} \iota_{A_{i}}(d f \wedge \phi)-\phi\left(A_{i}\right) d f\left(A_{i}\right)+f \hat{\delta}(\phi) \\
& =-\langle d f, \phi\rangle+f \hat{\delta}(\phi)
\end{aligned}
$$

since $\iota_{A_{i}} \iota_{A_{i}}(d f \wedge \phi)=0$.

Now we are in a position to deduce formulae for the induced differential operator in terms of the derivative flow $T X_{t}$.

2.5. A formula for the induced differential operator. We must now assume equation (2.1) is complete, which is to say $\zeta(x)=\infty$, almost surely. For a bounded smooth 1-form $\alpha$, suppose $\alpha_{t}$ satisfies

$$
\partial_{t} \alpha_{t}=\left(\operatorname{trace} \hat{\nabla}^{2}+\hat{\nabla}_{A_{0}}-\mathrm{Ric}_{A_{0}}-\hat{\nabla} A_{0}-\left(\hat{\nabla} A_{0}\right)^{*}+\operatorname{trace} \hat{\nabla} A_{0}\right) \alpha_{t}
$$

with $\alpha_{0}=\alpha$ and that $\Xi_{t}(x): T_{x} M \rightarrow T_{X_{t}(x)} M$ solves the covariant Itô equation

$$
\begin{aligned}
d^{\hat{\nabla}} \Xi_{t}(x)= & -\left(\operatorname{Ric}_{A_{0}}+\hat{\nabla} A_{0}+\left(\hat{\nabla} A_{0}\right)^{*}+\operatorname{trace} \hat{\nabla} A_{0}\right) \\
& \times\left(\Xi_{t}(x)\right) d t+\sum_{i=1}^{m} \breve{\nabla}_{\Xi_{t}(x)} A_{i} d B_{t}^{j}
\end{aligned}
$$

along the paths of $X_{t}(x)$ with $\Xi_{0}=\mathrm{id}_{T_{x} M}$. 
Fixing $T>0$, by Itô's formula we have

$$
\begin{aligned}
& d\left(\alpha_{T-t}\left(\Xi_{t}(x)\right)\right) \\
& =\sum_{i=1}^{m} \hat{\nabla}_{A_{i}} \alpha_{T-t}\left(\Xi_{t}(x)\right) d B_{t}^{i}+\hat{\nabla}_{A_{0}} \alpha_{T-t}\left(\Xi_{t}(x)\right) d t+\partial_{t} \alpha_{T-t}\left(\Xi_{t}(x)\right) d t \\
& \quad+\operatorname{trace} \hat{\nabla}^{2} \alpha_{T-t}\left(\Xi_{t}(x)\right) d t+\alpha_{T-t}\left(d^{\hat{\nabla}} \Xi_{t}(x)\right) \\
& =\sum_{i=1}^{m}\left(\left(\hat{\nabla}_{A_{i}} \alpha_{T-t}\right) \cdot+\alpha_{T-t}\left(\breve{\nabla} \cdot A_{i}\right)\right)\left(\Xi_{t}(x)\right) d B_{t}^{i} .
\end{aligned}
$$

It follows that $\alpha_{T-t}\left(\Xi_{t}(x)\right)$ is a local martingale, starting at $\alpha_{T}$. Furthermore, according to equation (26) in [6], for the derivative process $T X_{t}(x)$ we have

$$
d^{\hat{\nabla}} T X_{t}(x)=-\operatorname{Ric}\left(T X_{t}(x)\right) d t+\breve{\nabla}_{T X_{t}(x)} A_{0} d t+\sum_{i=1}^{m} \breve{\nabla}_{T X_{t}(x)} A_{i} d B_{t}^{i}
$$

and, therefore, by the variation of constants formula, we have

$$
\begin{aligned}
\Xi_{t}(x)= & T X_{t}(x) \\
& -T X_{t}(x) \int_{0}^{t} T X_{s}(x)^{-1}\left(\left(\hat{\nabla} A_{0}+\left(\hat{\nabla} A_{0}\right)^{*}+\operatorname{trace} \hat{\nabla} A_{0}\right)\left(\Xi_{s}(x)\right)\right) d s .
\end{aligned}
$$

Thus it is possible to calculate $\Xi_{t}(x)$ without using the parallel transport implicit in the original equation. Moreover, if the vector field $A_{0}$ vanishes then $\Xi_{t}(x)$ is given precisely by the derivative process $T X_{t}(x)$.

PROPOSITION 2.7. Suppose $h_{t}$ is an adapted process with paths in $L^{1,2}([0$, $T] ; \mathbb{R})$. Then

$$
\begin{aligned}
& \hat{\delta} \alpha_{T-t} h_{t}-\int_{0}^{t} h_{s} \alpha_{T-s}\left(A_{0}^{A}\right) d s \\
&+\frac{1}{2} \alpha_{T-t}\left(\Xi_{t}(x) \int_{0}^{t}\left(\dot{h}_{s}-\left(\operatorname{trace} \hat{\nabla} A_{0}\right)\left(X_{S}(x)\right) h_{s}\right)\right. \\
&\left.\Xi_{S}(x)^{-1} A\left(X_{S}(x)\right) d B_{S}\right)
\end{aligned}
$$

is a local martingale, starting at $\hat{\delta} \alpha_{T} h_{0}$, where the vector field $A_{0}^{A}$ is given by (2.15). 
ProOF. Set

$$
\mathbb{A}_{t}:=\exp \left(\int_{0}^{t}\left(\operatorname{trace} \hat{\nabla} A_{0}\right)\left(X_{s}(x)\right) d s\right)
$$

and define $\ell_{t}:=\mathbb{A}_{t}^{-1} h_{t}$. By equation (2.16), integration by parts and formula (2.12), we have, suppressing the summation over $i$, that

$$
\begin{aligned}
& d\left(\alpha_{T-t}\left(\Xi_{t}(x) \frac{1}{2} \int_{0}^{t} \mathbb{A}_{s} \dot{\ell}_{s} \Xi_{s}(x)^{-1} A\left(X_{s}(x)\right) d B_{s}\right)\right) \\
& \underline{\underline{m}} \frac{1}{2}\left(\left(\left(\hat{\nabla}_{A_{i}} \alpha_{T-t}\right) \cdot+\alpha_{T-t}\left(\breve{\nabla} \cdot A_{i}\right)\right)\left(\Xi_{t}(x)\right) d B_{t}^{i}\right) \\
& \times\left(\mathbb{A}_{t} \dot{\ell}_{t} \Xi_{t}(x)^{-1} A_{j}\left(X_{t}(x)\right) d B_{t}^{j}\right) \\
&=\left(\left(\hat{\nabla}_{A_{i}} \alpha_{T-t}\right) A_{i}+\alpha_{T-t}\left(\breve{\nabla}_{A_{i}} A_{i}\right)\right) \mathbb{A}_{t} \dot{\ell}_{t} d t \\
&=\left(\hat{\nabla}_{A_{i}} \alpha_{T-t}\right) A_{i} \mathbb{A}_{t} \dot{\ell}_{t} d t \\
&=-\left(\hat{\delta} \alpha_{T-t}\right) \mathbb{A}_{t} \dot{\ell}_{t} d t
\end{aligned}
$$

where $\stackrel{m}{=}$ denotes equality modulo the differential of a local martingale. By Proposition 2.5 and Itô's formula, we have

$$
d\left(\mathbb{A}_{t} \hat{\delta} \alpha_{T-t}\right) \stackrel{m}{=} \mathbb{A}_{t} \hat{\delta} \partial_{t} \alpha_{T-t} d t+\mathbb{A}_{t}\left(\mathscr{L}+\operatorname{trace} \hat{\nabla} A_{0}\right) \hat{\delta} \alpha_{T-t} d t=\mathbb{A}_{t} \alpha_{T-t}\left(A_{0}^{A}\right) d t
$$

which implies

$$
n_{t}:=\mathbb{A}_{t} \hat{\delta} \alpha_{T-t}-\int_{0}^{t} \mathbb{A}_{s} \alpha_{T-s}\left(A_{0}^{A}\right) d s
$$

is a local martingale, starting at $\hat{\delta} \alpha_{T}$. This implies

$$
\begin{aligned}
d\left(n_{t} \ell_{t}\right) & \stackrel{\mathrm{m}}{=} n_{t} \dot{\ell}_{t} d t \\
& =\left(\hat{\delta} \alpha_{T-t}\right) \mathbb{A}_{t} \dot{\ell}_{t} d t-\dot{\ell}_{t} \int_{0}^{t} \mathbb{A}_{s} \alpha_{T-s}\left(A_{0}^{A}\right) d s d t .
\end{aligned}
$$

Substituting the definition of $n_{t}$ into the left-hand side and performing integration by parts to the second term on the right-hand side implies

$$
\hat{\delta} \alpha_{T-t} h_{t}-\int_{0}^{t}\left(\hat{\delta} \alpha_{T-s}\right) \mathbb{A}_{s} \dot{\ell}_{s} d s-\int_{0}^{t} h_{s} \alpha_{T-s}\left(A_{0}^{A}\right) d s
$$

is another local martingale. Since

$$
\dot{\ell}_{t}=\mathbb{A}_{t}^{-1}\left(\dot{h}_{t}-\left(\operatorname{trace} \hat{\nabla} A_{0}\right)\left(X_{t}(x)\right) h_{t}\right),
$$

substituting formula (2.18) into the second term in (2.19) completes the proof. 
THEOREM 2.8. Suppose $h_{t}$ is any adapted process with paths in $L^{1,2}([0, \infty)$; $\mathbb{R})$ such that $h_{0}=0$ and $h_{T}=1$ and that $\alpha$ is a bounded smooth 1-form. Suppose (2.1) is complete and that the local martingales $\alpha_{T-t}\left(\Xi_{t}\right)$ and (2.17) are true martingales. Then

$$
\begin{aligned}
P_{T}(\hat{\delta} \alpha)=- & \frac{1}{2} \mathbb{E}\left[\alpha \left(\Xi _ { T } \int _ { 0 } ^ { T } \Xi _ { t } ^ { - 1 } \left(\left(\dot{h}_{t}-\left(\operatorname{trace} \hat{\nabla} A_{0}\right)\left(X_{t}\right) h_{t}\right) A\left(X_{t}\right) d B_{t}\right.\right.\right. \\
& \left.\left.\left.+2 h_{t} A_{0}^{A} d t\right)\right)\right] .
\end{aligned}
$$

PROOF. By (2.16), we have

$$
\alpha_{T-t}\left(\Xi_{t}\right)=\alpha\left(\Xi_{T}\right)-\int_{t}^{T}\left(\left(\hat{\nabla}_{A_{i}} \alpha_{T-s}\right) \cdot+\alpha_{T-s}\left(\breve{\nabla} \cdot A_{i}\right)\right)\left(\Xi_{t}\right) d B_{t}^{i}
$$

and, therefore,

$$
\begin{aligned}
& \mathbb{E}\left[\int_{0}^{T} \alpha_{T-t}\left(\Xi_{t} h_{t} \Xi_{t}^{-1} A_{0}^{A}\right) d t\right] \\
& \quad=\mathbb{E}\left[\alpha\left(\Xi_{T} \int_{0}^{T} h_{t} \Xi_{t}^{-1} A_{0}^{A} d t\right)\right]
\end{aligned}
$$

since $\alpha_{T-t}\left(\Xi_{t}\right)$ is assumed to be a martingale. The result now follows from Proposition 2.7 , by taking expectations.

In analogy with Lemma 1.14, an integrability assumption on $h$ plus suitable bounds on $\hat{\nabla} A_{0}$, trace $\hat{\nabla} A_{0}, A_{0}^{A}$ and $\hat{\delta} \alpha$ and on the moments of $T X_{t}$ and $T X_{t}^{-1}$ would be sufficient to guarantee that $\alpha_{T-t}\left(\Xi_{t}\right)$ and (2.17) are true martingales.

Corollary 2.9. Suppose $f$ is a bounded smooth function. Suppose $V$ is a bounded smooth vector field with $\sum_{i=1}^{m} A_{i}\left\langle V, A_{i}\right\rangle$ bounded. Then, under the assumptions of Theorem 2.8 with $\alpha=f V^{b}$, we have

$$
\begin{aligned}
& P_{T}(V(f)) \\
& =-\sum_{i=1}^{m} \mathbb{E}\left[f\left(X_{T}\right) A_{i}\left\langle V, A_{i}\right\rangle\left(X_{T}\right)\right] \\
& \quad+\frac{1}{2} \mathbb{E}\left[f ( X _ { T } ) \left\langleV, \Xi_{T} \int_{0}^{T} \Xi_{t}^{-1}\left(\left(\dot{h}_{t}-\left(\operatorname{trace} \hat{\nabla} A_{0}\right)\left(X_{t}\right) h_{t}\right) A\left(X_{t}\right) d B_{t}\right.\right.\right. \\
& \left.\left.\left.\quad+2 h_{t} A_{0}^{A} d t\right)\right\rangle\right]
\end{aligned}
$$


with

$$
\begin{gathered}
\Xi_{t}=T X_{t}-T X_{t} \int_{0}^{t} T X_{s}^{-1}\left(\left(\hat{\nabla} A_{0}\right)^{*}+\hat{\nabla} A_{0}+\operatorname{trace} \hat{\nabla} A_{0}\right)\left(\Xi_{s}\right) d s, \\
A_{0}^{A}=\sum_{i=1}^{m}\left(\left(\hat{\nabla} A_{0}\right)^{*}+\hat{\nabla} A_{0}\right)\left(\breve{T}\left(\cdot, A_{i}\right)^{*}\left(A_{i}\right)\right)+\left[A_{0}, \breve{T}\left(\cdot, A_{i}\right)^{*}\left(A_{i}\right)\right],
\end{gathered}
$$

where the operators $\hat{\nabla} A_{0}$ and $\breve{T}\left(\cdot, A_{i}\right)$ are given at each $x \in M$ and $v \in T_{x} M$ by

$$
\begin{aligned}
\hat{\nabla}_{v} A_{0} & =A(x)\left(d\left(A^{*}(\cdot) A_{0}(\cdot)\right)_{x}(v)-\left(d A^{*}\right)_{x}\left(v, A_{0}\right)\right), \\
\breve{T}\left(v, A_{i}\right)_{x} & =A(x)\left(d A^{*}\right)_{x}\left(v, A_{i}\right) .
\end{aligned}
$$

PROOF. This follows from Theorem 2.8. In particular, Lemma 2.6 implies

$$
V(f)=f \hat{\delta}\left(V^{b}\right)-\hat{\delta}\left(f V^{b}\right)
$$

while formula (2.13), the Le Jan-Watanabe property and the adaptedness of $\breve{\nabla}$ imply

$$
\hat{\delta}\left(V^{b}\right)=-\sum_{i=1}^{m}\left\langle\breve{\nabla}_{A_{i}} V, A_{i}\right\rangle=-\sum_{i=1}^{m} A_{i}\left\langle V, A_{i}\right\rangle
$$

Note that if (2.1) is a gradient system then $\mathscr{L}=\Delta+A_{0}$ and $A_{0}^{A}$ vanishes and

$$
\sum_{i=1}^{m} A_{i}\left\langle V, A_{i}\right\rangle=\operatorname{div} V .
$$

In this case, since trace $\hat{\nabla} A_{0}=\operatorname{div} A_{0}$, Corollary 2.9 yields the unfiltered version of Corollary 1.17.

COROLLARY 2.10. Under the assumptions of Corollary 2.9, we have

$$
\begin{aligned}
\left(d \log p_{T}(x, \cdot)\right)_{y}(v) & \\
= & -\left\langle v, \sum_{i=1}^{m} \breve{T}\left(\cdot, A_{i}\right)^{*}\left(A_{i}\right)(y)\right\rangle \\
& -\frac{1}{2}\left\langle v, \mathbb{E}\left[\Xi _ { T } \int _ { 0 } ^ { T } \Xi _ { t } ^ { - 1 } \left(\left(\dot{h}_{t}-\left(\operatorname{trace} \hat{\nabla} A_{0}\right)\left(X_{t}\right) h_{t}\right) A\left(X_{t}\right) d B_{t}\right.\right.\right. \\
& \left.\left.\left.+2 h_{t} A_{0}^{A} d t\right) \mid X_{T}(x)=y\right]\right\rangle
\end{aligned}
$$

for all $v \in T_{y} M$ where the various terms appearing in the right-hand side can be calculated as in Corollary 2.9. 
PROOF. Since Corollary 2.9 holds for all smooth functions $f$ and vector fields $V$ of compact support, and since by Lemma 2.6

$$
f \hat{\delta}\left(V^{b}\right)-\hat{\delta}\left(f V^{b}\right)=V(f)=f \delta\left(V^{b}\right)-\delta\left(f V^{b}\right),
$$

the result follows from equation (2.14), Lemma 2.4 and Corollary 2.9.

EXAMPLE 2.11. Consider the special case in which $M=\mathbb{R}^{n}$. Denote by $q_{T}(x, y)$ the smooth density of $X_{T}(x)$ with respect to the standard $n$-dimensional Lebesgue measure. Recall that $p_{T}(x, y)$ denotes the density with respect to the induced Riemannian measure. It follows that

$$
q_{T}(x, y)=p_{T}(x, y) \rho^{1 / 2}(y),
$$

where $\rho(y)$ denotes the absolute value of the determinant of the matrix

$$
\left\{\left\langle A^{*} \partial_{i}, A^{*} \partial_{j}\right\rangle_{\mathbb{R}^{m}}(y)\right\}_{i, j=1}^{n}
$$

in which $\left\{\partial_{i}\right\}_{i=1}^{n}$ denotes the standard basis of vector fields on $\mathbb{R}^{n}$. Consequently,

$$
\left(d \log q_{T}(x, \cdot)\right)_{y}(v)=\left(d \log p_{T}(x, \cdot)\right)_{y}(v)+\left(d \log \rho^{1 / 2}(\cdot)\right)_{y}(v)
$$

with the first term on the right-hand side given, in terms of the induced metric, by Corollary 2.10 .

\section{REFERENCES}

[1] Bismut, J.-M. (1984). Large Deviations and the Malliavin Calculus. Progress in Mathematics 45. Birkhäuser, Boston, MA. MR0755001

[2] Cruzeiro, A. B. and ZHANG, X. (2006). Bismut type formulae for diffusion semigroups on Riemannian manifolds. Potential Anal. 25 121-130. MR2238939

[3] Driver, B. K. and Thalmaier, A. (2001). Heat equation derivative formulas for vector bundles. J. Funct. Anal. 183 42-108. MR1837533

[4] Elworthy, K. D., LE JAn, Y. and LI, X.-M. (1999). On the Geometry of Diffusion Operators and Stochastic Flows. Lecture Notes in Math. 1720. Springer, Berlin. MR1735806

[5] Elworthy, K. D., LE JAn, Y. and Li, X.-M. (2010). The Geometry of Filtering. Frontiers in Mathematics. Birkhäuser, Basel. MR2731662

[6] Elworthy, K. D., LE JAN, Y. and Li, X.-M. (1997). Concerning the geometry of stochastic differential equations and stochastic flows. In New Trends in Stochastic Analysis (Charingworth, 1994) 107-131. World Scientific, River Edge, NJ.

[7] Elworthy, K. D. and Li, X.-M. (1994). Formulae for the derivatives of heat semigroups. J. Funct. Anal. 125 252-286. MR1297021

[8] Elworthy, K. D. and Li, X.-M. (1998). Bismut type formulae for differential forms. C. R. Acad. Sci. Paris Sér. I Math. 327 87-92. MR1650216

[9] ENGEL, K.-J. and NAGEL, R. (2000). One-Parameter Semigroups for Linear Evolution Equations. Graduate Texts in Mathematics 194. Springer, New York. MR1721989

[10] Kobayashi, S. and NomizU, K. (1963). Foundations of Differential Geometry. Vol I. Wiley, New York-London. MR0152974

[11] Kunita, H. (1990). Stochastic Flows and Stochastic Differential Equations. Cambridge Studies in Advanced Mathematics 24. Cambridge Univ. Press, Cambridge. MR1070361 
[12] Nakahara, M. (2003). Geometry, Topology and Physics, 2nd ed. Graduate Student Series in Physics. Institute of Physics, Bristol. MR2001829

[13] ShigeKaWA, I. (1982). On stochastic horizontal lifts. Z. Wahrsch. Verw. Gebiete 59 211-221. MR0650613

[14] Stroock, D. W. (2000). An Introduction to the Analysis of Paths on a Riemannian Manifold. Mathematical Surveys and Monographs 74. Amer. Math. Soc., Providence, RI. MR1715265

[15] Thalmaier, A. (1997). On the differentiation of heat semigroups and Poisson integrals. Stoch. Stoch. Rep. 61 297-321. MR1488139

[16] Thalmaier, A. and WANG, F.-Y. (1998). Gradient estimates for harmonic functions on regular domains in Riemannian manifolds. J. Funct. Anal. 155 109-124. MR1622800

[17] Thalmaier, A. and WANG, F.-Y. (2011). A stochastic approach to a priori estimates and Liouville theorems for harmonic maps. Bull. Sci. Math. 135 816-843. MR2838103

[18] WANG, F.-Y. (2014). Integration by parts formula and shift Harnack inequality for stochastic equations. Ann. Probab. 42 994-1019. MR3189064

[19] WANG, F.-Y. (2014). Analysis for Diffusion Processes on Riemannian Manifolds. Advanced Series on Statistical Science \& Applied Probability 18. World Scientific, Hackensack, NJ. MR3154951

\author{
Mathematics Research UNit \\ UNIVERSITY OF LUXEMBOURG \\ CAMPus BElVAL-MAison DU NOMBRE \\ 6, AVENUE DE LA FONTE \\ L-4364 ESCH-SUR-ALZETTE \\ GRAND DUCHY OF LUXEMBOURG \\ E-MAIL: anton.thalmaier@uni.lu \\ james.thompson@uni.lu
}

